\title{
Article
}

\section{Nematic Dispersive Shock Waves from Nonlocal to Local}

\author{
Saleh Baqer $1,+\left(\mathbb{D}\right.$, Dimitrios J. Frantzeskakis $2,+\left(\mathbb{D}\right.$, Theodoros P. Horikis ${ }^{3,+}+\mathbb{C}$, Côme Houdeville ${ }^{4,+}$, \\ Timothy R. Marchant ${ }^{5,6,+} \mathbb{D}$ and Noel F. Smyth ${ }^{7, *,+} \mathbb{D}$ \\ 1 Department of Mathematics, Faculty of Science, Kuwait University, Kuwait City 13060, Kuwait; \\ saleh.baqer@ku.edu.kw \\ 2 Department of Physics, National and Kapodistrian University of Athens, 15784 Athens, Greece; \\ dfrantz@phys.uoa.gr \\ 3 Department of Mathematics, University of Ioannina, 45110 Ioannina, Greece; horikis@uoi.gr \\ 4 Ecole Nationale Supérieure de Techniques Avancées, 828 Boulevard des Maréchaux, Palaiseau, \\ 91120 Paris, France; come.houdeville@ensta-paris.fr \\ 5 Australian Mathematical Sciences Institute, University of Melbourne, Melbourne, VIC 3052, Australia; \\ t.marchant@uow.edu.au \\ 6 School of Mathematics and Applied Statistics, University of Wollongong, Northfields Avenue, \\ Wollongong, NSW 2522, Australia \\ 7 School of Mathematics, University of Edinburgh, Edinburgh EH9 3FD, UK \\ * Correspondence: N.Smyth@ed.ac.uk \\ + All authors contributed equally to this work.
}

check for updates

Citation: Baqer, S.; Frantzeskakis, D.J.; Horikis, T.P.; Houdeville, C.; Marchant, T.R.; Smyth, N.F. Nematic Dispersive Shock Waves from Nonlocal to Local. Appl. Sci. 2021, 11, 4736. https://doi.org/10.3390/ app11114736

Academic Editor: Andrés Márquez

Received: 4 May 2021

Accepted: 18 May 2021

Published: 21 May 2021

Publisher's Note: MDPI stays neutral with regard to jurisdictional claims in published maps and institutional affiliations.

Copyright: (c) 2021 by the authors. Licensee MDPI, Basel, Switzerland. This article is an open access article distributed under the terms and conditions of the Creative Commons Attribution (CC BY) license (https:/ / creativecommons.org/licenses/by/ $4.0 /)$.

\begin{abstract}
The structure of optical dispersive shock waves in nematic liquid crystals is investigated as the power of the optical beam is varied, with six regimes identified, which complements previous work pertinent to low power beams only. It is found that the dispersive shock wave structure depends critically on the input beam power. In addition, it is known that nematic dispersive shock waves are resonant and the structure of this resonance is also critically dependent on the beam power. Whitham modulation theory is used to find solutions for the six regimes with the existence intervals for each identified. These dispersive shock wave solutions are compared with full numerical solutions of the nematic equations, and excellent agreement is found.
\end{abstract}

Keywords: nematic liquid crystal; dispersive shock wave; solitary wave; soliton; modulation theory

\section{Introduction}

Nematic liquid crystals form an ideal medium to study nonlinear optics due to their "huge" nonlinearity, which is orders of magnitude larger than that of optical fibers, so that nonlinear effects can be observed over millimeter distances [1-4]. In particular, the refractive index of nematic liquid crystals increases with optical intensity, so that they form a focusing medium. When a light beam propagates through a nematic liquid crystal, the electric field of the electromagnetic wave induces dipoles in the nematic molecules, which then rotate, changing the refractive index. In addition, nematic liquid crystals have a nonlocal response to an optical beam in that the elastic response of the nematic extends far beyond the optical forcing [4]. An optical beam propagating through a nematic medium can then form its own waveguide, resulting in a self-guided beam, an optical solitary wave, termed a "nematicon" $[2,3,5,6]$, which was first experimentally generated and observed in 2000 [6]. Since this first observation, nematicons, and related solitary-type waves, such as optical vortices, have become a theme of intense experimental and theoretical research effort, driven both by interest in the nonlinear optics of nematic liquid crystals and also by their potential applications in optical devices [7-10]; see Refs. [2,3,5,11] for general reviews on the nonlinear optics of nematic liquid crystals.

Solitary waves are generic wave forms for nonlinear dispersive wave equations [12], first observed and studied in the context of water waves [12,13] and fluid dynamics [12], but are widespread in nature arising, e.g., in solid mechanics [14], biology [15], ecology [16], 
and the aforementioned context of nonlinear optics [2,17,18], for instance. One of the appealing features of solitary waves, in addition to their widespread occurrence in nature, is that they are localized waves with steady profiles, which makes them easier to study theoretically. In addition to this, many generic nonlinear dispersive wave equations, such as the Korteweg-de Vries (KdV), nonlinear Schrödinger (NLS), and Sine-Gordon equations, are completely integrable systems via the Inverse Scattering Transform method [12,18]. Thus, a general initial condition for these equations will form a finite number of solitary waves, plus dispersive radiation. In addition, solitary wave solutions of integrable nonlinear dispersive wave equations, solitons, interact "elastically", i.e., they emerge unscathed out of the interaction without any change in their form; hence, due to this particle-like behavior, solitary waves are termed solitons for such equations.

Another generic wave form supported by nonlinear dispersive wave equations are dispersive shock waves (DSWs), also termed undular bores; these structures are as widespread in nature as solitary waves, with well known examples being tidal bores and tsunamis [19]. In contrast to a solitary wave, a DSW is a non-steady wave form which continuously expands. A DSW is a dispersive regularization of a discontinuity and is a modulated periodic wavetrain with solitary waves at one edge and linear, dispersive waves at the other-see Reference [19] for a general review of DSWs. Since DSWs are non-steady waveforms, their study is more difficult than that for solitary waves. The development of DSW solutions of nonlinear dispersive wave equations relies chiefly on Whitham modulation theory [12,20-22], which is a version of the asymptotic method of multiple scales that is used to analyze slowly varying periodic wavetrains. Whitham modulation equations are a system of partial differential equations which govern the parameters of a slowly varying wavetrain, such as its amplitude, wavenumber, frequency, and mean height. If this system is hyperbolic, then the underlying wavetrain is modulationally stable, while, if it is elliptic, the wavetrain is unstable [12]. A major achievement of Whitham modulation theory was the development of the modulation equations for the KdV equation [12,21]. These modulation equations form a hyperbolic system, so that the cnoidal wave solution of the $\mathrm{KdV}$ equation is modulationally stable. It was subsequently realized that a simple wave solution of the KdV modulation equations is a DSW [23], even though the initial condition is a step, which is not slowly varying. This DSW solution is in excellent agreement with numerical solutions of the KdV equation [24]. The key to the determination of the simple wave DSW solution is the ability to set the modulation equations in Riemann invariant form. If the nonlinear dispersive wave equation governing the DSW is integrable, then its Whitham modulation equations can be automatically set in Riemann invariant form [25], so that the DSW solution can easily be found.

As mentioned above, the standard DSW form, termed of KdV type [19], is a modulated periodic wave with solitary waves at one edge and linear dispersive waves at the other. A non-standard DSW type is a resonant DSW [26,27], for which the waves of the DSW are in resonance with (linear) dispersive waves, resulting in a resonant wavetrain being emitted from the DSW. Resonant DSWs also occur for the KdV equation with next higher-order dispersion, i.e., fifth-order dispersion, namely for the Kawahara equation [28], and the NLS equation with next order, third-order dispersion [29-32]. If the emitted resonant wavetrain is of large enough amplitude, the KdV-type DSW structure can be destroyed; this results in the so-called traveling dispersive shock wave (TDSW) regime [26,27], consisting of a resonant wavetrain with a negative polarity solitary wave, which is the remnant of the DSW, linking this to the level behind [26] - see Figure $2 \mathrm{~d}$ below for an example of such a TDSW. A nematic liquid crystal is a focusing medium; thus, optical waves are modulationally unstable; as a result, an optical DSW is not supported. However, the addition of azo dyes to the nematic medium changes its response so that it becomes defocusing [33]; in this case, nematics can support DSWs [34-36]. A nematic DSW is an example of a resonant DSW [34-36]. In these works, the nematic DSW was studied in the highly nonlocal limit, for which the nematic elastic response extends far beyond the light beam, with the nematic DSW generated by a step jump in the optical intensity. While the nematic equations are 
of NLS-type [2], in the highly nonlocal limit the nematic bore is of KdV-type and is well described by the DSW solution of the KdV equation. The nematic DSW structure is highly dependent on the size of the jump of the optical electric field intensity generating it, with six distinct DSW types identified [36].

As stated above, in the highly nonlocal limit the nematic DSW is of KdV-type with the DSW having positive polarity. However, in the limit of weak nonlocality, the nematic equations reduce to the NLS equation [2,4], and the nematic DSW becomes the NLS DSW, which is non-resonant. The degree of nonlocality of the optical response of a nematic is inversely proportional to the power of the optical beam, with the response being highly nonlocal for lower power beams, transitioning to local as the beam power increases [2,4], as will be detailed in Section 2. In this work, the evolution of the nematic DSW structure as the degree of nonlocality ranges from highly nonlocal (low power beams) [34-36] to local (high power beams) will be studied. As the nonlocality decreases, the changes in the DSW structure from those previously found [36] in the limit of high nonlocality to the standard NLS DSW [37] will be identified, and the solutions for these will be derived. It is found that there exist two additional DSW regimes over those for large nonlocality, including the NLS DSW for zero nonlocality. The new DSW regime is a transition between the KdV DSW behavior for large nonlocality and the NLS DSW behavior for very small nonlocality. In this regime, the DSW structure consists of a resonant wavetrain headed by a partial DSW which takes the solution to the initial level ahead, similar to the resonant DSW for the $\mathrm{KdV}$ equation with fifth-order dispersion $[27,38]$. As the nonlocality decreases, the optical power increases, the resonant wavetrain contracts with the leading partial DSW expanding and becoming a full NLS DSW. The analytical solutions for the various DSW types will be compared with full numerical solutions of the nematic equations.

\section{Nematic Equations}

Let us consider the propagation of a linearly, extra-ordinarily polarized, coherent light beam of wavenumber $k_{0}$, wavelength $\lambda_{0}=2 \pi / k_{0}$, through a planar cell filled with nematic liquid crystals. The optical beam is assumed to propagate down the cell along in the $Z$ direction, with its electric field $E$ polarized in the $Y$ direction. The coordinate $X$ then completes the coordinate system. Nematic liquid crystals are a uniaxial medium consisting of elongated molecules, with the long axis termed the molecular director. The refractive index of the medium is $n_{\|}$for optical beams polarized along the molecular director and $n_{\perp}$ for fields polarized orthogonal to the director. A fundamental property of nematic liquid crystals is the so-called Freédericksz threshold, whereby a minimum optical power is needed to rotate the nematic molecules, thus changing the refractive index of the medium [1]. However, high optical powers lead to heating of the nematic medium, which can cause the loss of the nematic state if the temperature change is high enough [1,6]. One method to overcome this is to pre-tilt the nematic molecules at an angle $\theta_{0}$ with respect to the $Z$-direction upon the application of an external static electric field $E_{L F}$, so that milli-Watt power beams can rotate the nematic molecules [6]. Let us denote the optically induced rotation of the nematic by $\phi$, so that, in the presence of an optical beam, the total angle of the nematic director to the $Z$ direction is $\theta=\theta_{0}+\phi$. This configuration of the nematic cell is illustrated in Figure 1. The dimensional equations governing the propagation of the optical beam in the nematic cell are then of the following form:

$$
2 i k_{0} n_{e} \frac{\partial E}{\partial Z}+\nabla^{2} E+k_{0}^{2}\left[n_{\perp}^{2} \cos ^{2} \theta+n_{\|}^{2} \sin ^{2} \theta-n_{\perp}^{2} \cos ^{2} \theta_{0}-n_{\|}^{2} \sin ^{2} \theta_{0}\right] E=0,
$$

for the electric field of the beam, and

$$
K \nabla^{2} \phi+\left[\frac{1}{4} \epsilon_{0} \Delta \epsilon|E|^{2}+\frac{1}{2} \Delta \epsilon_{L F} E_{L F}^{2}\right] \sin 2\left(\theta_{0}+\phi\right)=0,
$$


for the nematic response [2-5]. Here, the extraordinary refractive index of the nematic is:

$$
n_{e}^{2}=\frac{n_{\perp}^{2} n_{\|}^{2}}{n_{\|}^{2} \cos ^{2} \theta+n_{\perp}^{2} \sin ^{2} \theta} .
$$

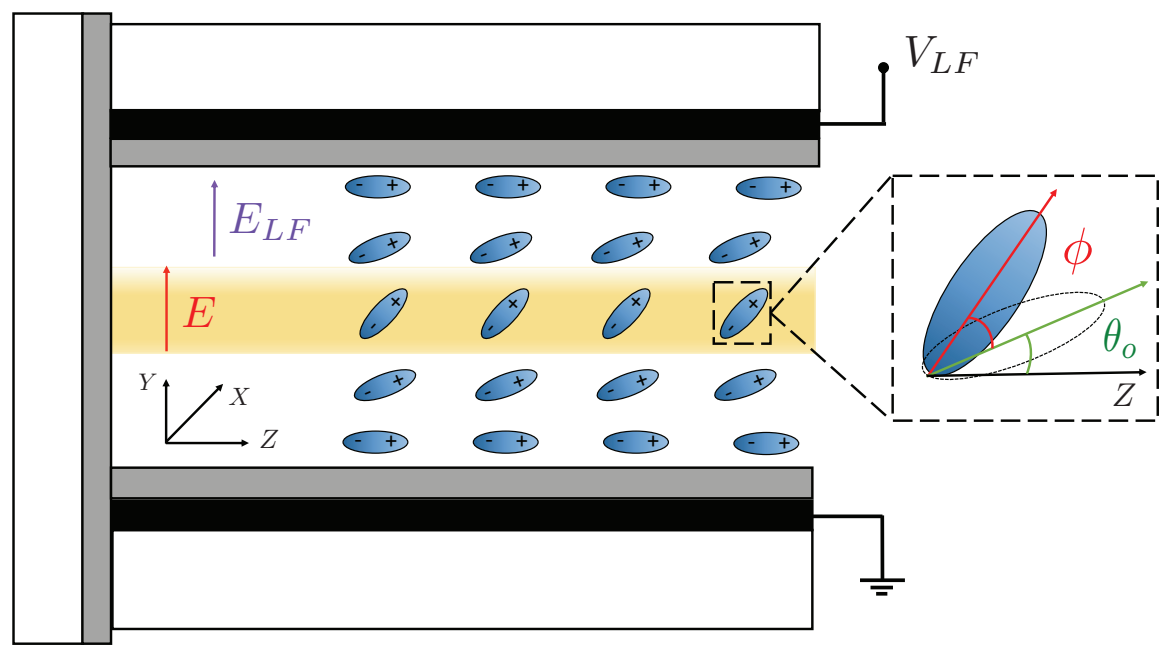

Figure 1. Sketch of nematic cell. A coherent light beam (yellow region) in which the electric field $E$ is polarized in the $Y$ direction propagates in the $Z$ direction through a cell filled with a dye-doped nematic liquid crystal. Thin film electrodes (black) are deposited on the upper and lower cell walls (grey). An external low frequency voltage bias $V_{L F}$ creates an electric field $E_{L F}$ to pre-tilt the molecules at an angle $\theta_{0}$ to $Z$. The nematic molecules which are located at the boundaries are held tightly by the virtue of the anchoring films. The far right inset (black) dashed box exhibits the angular rotation of a nematic molecule with respect to the propagation direction $Z$ in the absence $\left(\theta_{0}\right)$ and presence $\left(\theta_{0}+\phi\right)$ of the optical beam.

In the above equations, $\Delta \epsilon=n_{\|}^{2}-n_{\perp}^{2}$ is the optical anisotropy, $\Delta \epsilon_{L F}$ is the lowfrequency dielectric anisotropy, and $\epsilon_{0}$ is the electrical permittivity of free space. In addition, the constant $K$ is the elastic medium constant in the one constant approximation for which the elastic constants of bend, twist, and splay are taken equal [1,2].

The nematic Equations (1) and (2) are highly nonlinear and difficult to analyze. However, for milli-Watt power beams, the optically induced rotation $\phi$ is small, $|\phi| \ll \theta_{0}$, so that these equations can be expanded in Taylor series around $\theta_{0}$. In addition, these equations can be put in dimensionless form using typical scales $L_{Z}$ down the cell and $W$ transverse to the down cell direction, as well as a typical scale $A_{b}$ for the electric field of the optical beam, so that

$$
Z=L_{z} z, \quad X=W x, \quad Y=W y, \quad E=A_{b} u .
$$

Here, $(x, y, z)$ is the non-dimensional coordinate system, and $u$ is the non-dimensional electric field of the optical beam. The electric field scale is obtained by assuming that the input optical beam is a Gaussian beam of power $P_{b}$, amplitude $A_{b}$, and width $W_{b}$, so that

$$
A_{b}^{2}=\frac{2 P_{b}}{\pi \Gamma W_{b}^{2}}, \quad \Gamma=\frac{1}{2} \epsilon_{0} c n_{e}, \quad n_{e}^{2}=\frac{n_{\|}^{2} n_{\perp}^{2}}{n_{\|}^{2} \cos ^{2} \theta_{0}+n_{\perp}^{2} \sin ^{2} \theta_{0}} .
$$

Substituting these into the nematic Equations (1) and (2), and expanding in Taylor series for small $|\phi|$, we find $[5,39]$ that suitable scalings are

$$
L_{Z}=\frac{4 n_{e}}{\Delta \epsilon k_{0} \sin 2 \theta_{0}}, \quad W=\frac{2}{k_{0} \sqrt{\Delta \epsilon \sin 2 \theta_{0}}},
$$


and the resulting non-dimensional equations read

$$
\begin{aligned}
i \frac{\partial u}{\partial z}+\frac{1}{2} \nabla^{2} u+2 \phi u & =0, \\
v \nabla^{2} \phi-2 q \phi & =-2|u|^{2} .
\end{aligned}
$$

Here, the dimensionless elasticity and pre-tilting parameters, $v$ and $q$, are given by

$$
v=\frac{8 K}{\epsilon_{0} \Delta \epsilon A_{b}^{2} W^{2} \sin 2 \theta_{0}}=\frac{\pi K \Gamma k_{0}^{2} W_{b}^{2}}{\epsilon_{0} P_{b}}, \quad q=\frac{4 \Delta \epsilon_{L F} E_{L F}^{2} \cos 2 \theta_{0}}{\epsilon_{0} \Delta \epsilon A_{b}^{2} \sin 2 \theta_{0}} .
$$

Typical experimental beam parameter values are power $P_{b}=2 \mathrm{~mW}$ and half-width $W_{b}=1.5 \mu \mathrm{m}$, with a wavelength $\lambda_{0}=2 \pi / k_{0}=1.064 \mu \mathrm{m}$ in the near infrared $[2,5]$. For the liquid crystal E7, a typical elastic constant is $K=1.2 \times 10^{-11} N$. These parameter values give the elasticity parameter $v=O(100)$, as found in other studies $[5,40,41]$. This high value of $v$ means that the nematic is operating in the highly nonlocal regime, in that the elastic response of the nematic extends far beyond the waist of the optical beam [2-4]. However, $v$ is inversely proportional to the beam power $P_{b}$. Note that, for $v=0$, the nematic Equations (7) and (8) reduce to the standard NLS equation

$$
i \frac{\partial u}{\partial z}+\frac{1}{2} \nabla^{2} u+\frac{2}{q}|u|^{2} u=0 .
$$

This is the local response limit for the nematic. Note that, in $(2+1)$-dimensions, beams governed by this equation are unstable and can show catastrophic collapse above a critical power [17]. It is known that a nonlocal response, $v$ large, stabilizes $(2+1)$-dimensional optical beams [2-5]. This is because the nematic response Equation (2) is elliptic, so its solution depends on $u$ in the entire domain, the origin of the physical concept of nonlocality. Hence, by adjusting the beam power $P_{b}$, the response of the medium can be adjusted from nonlocal to local, as long as the induced heating does not destroy the nematic phase at high power.

The nematic system (7) and (8) is a focusing NLS-type system, that is the refractive index in the dimensional Equation (1) increases with beam intensity $|u|^{2}$. Since focusing NLS equations do not possess (stable) DSW solutions, the equation needs to be defocusing; in such a case, the refractive index decreases with beam intensity, and DSW solutions do exist [19]. The nematic medium can feature a defocusing response through the addition of azo-dyes [33]. The change in the nematic response due to the addition of the azo-dye is physically complicated, with the "order parameter" change being opposite to that in the absence of the dye. A simple model of this response change is to modify the electric field Equation (7) from focusing to defocusing. In addition, the analysis of DSWs is simplest in $(1+1)$-dimensions as then there are no geometric spreading effects. With these assumptions and simplifications, the nematic equations become

$$
\begin{aligned}
i \frac{\partial u}{\partial z}+\frac{1}{2} \frac{\partial^{2} u}{\partial x^{2}}-2 \phi u & =0 \\
v \frac{\partial^{2} \phi}{\partial x^{2}}-2 q \phi & =-2|u|^{2}
\end{aligned}
$$

The same system of equations also describes optical beam propagation in thermal optical media for which the refractive index depends on the temperature of the medium [42]. Such thermal optical media typically have a defocusing response. For these defocusing nematic equations, a suitable initial condition which will generate a DSW is the intensity jump initial condition 


$$
u=\left\{\begin{array}{ll}
u_{-}, & x<0 \\
u_{+}, & x>0
\end{array}, \quad \phi=\left\{\begin{array}{cc}
\frac{u_{-}^{2}}{q}, & x<0 \\
\frac{u_{+}^{2}}{q}, & x>0
\end{array} .\right.\right.
$$

The DSW solution of the defocusing nematic Equations (11) and (12) has been studied in the highly nonlocal limit $v \gg 1$ in previous work [34-36]. As stated above, in the local limit $v \ll 1$, this system reduces to a perturbed defocusing NLS equation and becomes the defocusing NLS equation for $v=0$. The DSW solution of the defocusing NLS equation is known [37], so that the perturbed local DSW solution can be found using perturbed Whitham modulation theory [43]. Before studying the behavior of the nematic DSW as $v$ varies from large to small, some previously derived results [34-36] will be briefly summarized.

The analytical DSW solutions derived in this work will be compared with full numerical solutions of the nematic Equations (11) and (12) with the initial condition (13). The electric field Equation (11) was solved using the pseudo-spectral method of Fornberg and Whitham [24], as extended [44,45] to improve the stability for high wavenumbers through the use of an integrating factor. The $x$ derivatives were calculated using the Fast Fourier Transform (FFT), and the solution was advanced in $z$ in Fourier space employing the fourth-order Runge-Kutta method, as detailed in previous work $[36,46]$. The step initial condition (13) was smoothed using the hyperbolic tangent function, as detailed in Reference [36,46]. The director Equation (12) was also solved using the FFT, as the equation does not have a singularity at zero wavenumber due to the $2 q \theta$ term.

Figure 2 displays a summary of the nematic DSW types as the nonlocality $v$ varies from large $v$, corresponding to a highly nonlocal response for low beam power, to small $v$, pertinent to a local medium response for high beam power. The terminology for the DSW regimes will be taken from previous work on radiating DSWs [26,36]. In particular, the DSW regimes from nonlocal to local response will now be detailed, for $u_{-}=1$ and $u_{+}=0.8$, with the existence intervals for the various DSW types given in Table 1.

- $\quad$ PDSW (perturbed DSW): This regime is illustrated in Figure 2a. The nematic DSW is essentially a KdV DSW governed by the Kawahara Equation (44) and its solution can be found as a perturbed KdV DSW [36,47]. This DSW regime is non-resonant and there is no resonant wavetrain attached to the leading edge of the DSW.

- $\quad$ RDSW (radiating DSW): As the nonlocality $v$ decreases, the DSW becomes resonant with a resonant wavetrain attached to the leading edge of the DSW, as illustrated in Figure $2 b$. As all individual waves in the DSW are resonant, resonant waves are emitted from the DSW, which results in the DSW not being rank ordered [36].

- $\quad$ CDSW (crossover DSW): As the nonlocality decreases and the amplitude of the resonant wavetrain grows, the DSW becomes unstable with a total loss of the rank ordering of the waves of the DSW [26,36]. This regime is illustrated in Figure 2c.

- TDSW (traveling DSW): As the amplitude of the resonant wavetrain grows, the shedding of conserved quantities into resonant radiation eventually destroys the DSW, leaving a high amplitude resonant wavetrain with a negative polarity solitary wave linking this wavetrain to the intermediate level [26] as seen in Figure $2 \mathrm{~d}$. While there is a solitary wave linking the resonant wavetrain to the intermediate level, this linking can be conveniently treated as a Whitham shock [48], a shock wave in the Whitham modulation equation variables. The resonant wavetrain is brought down to the level $u_{+}$ahead by a partial DSW [36]. This partial DSW has linear dispersive waves at its leading edge but has a finite wavelength wave at its trailing edge, that is, it is not bounded by solitary waves at the trailing edge [38,49], as for a standard DSW.

- $\quad$ RNLS DSW (radiating NLS DSW): Further decrease in the nonlocality results in the amplitude of the linking solitary wave becoming negligible, so that the wave form consists of a (stable) resonant wavetrain headed by a partial DSW which brings the wavetrain down to the level $u_{+}$ahead, as seen in Figure 2e. This DSW regime does not occur in the high nonlocality limit as it is a "bridge" to the local NLS DSW for $v=0$. 
- NLS DSW: As the nonlocality $v$ decreases to $v=0$ and the nematic Equations (11) and (12) reduce to the NLS equation, the resonant wavetrain length contracts and the leading partial DSW evolves to a full DSW, with linear dispersive waves at the leading edge and solitary waves at the trailing edge. The resonant wavetrain then disappears and the leading DSW bringing the wavetrain down to the level $u_{+}$ahead attaches to the intermediate level with $|u|=u_{i}$, as in Figure 2f. The resulting DSW is essentially an NLS DSW, which completes the transition from the KdV-type DSW for high nonlocality, that is for low power beams, to a NLS DSW for low nonlocality, that is for high power beams.
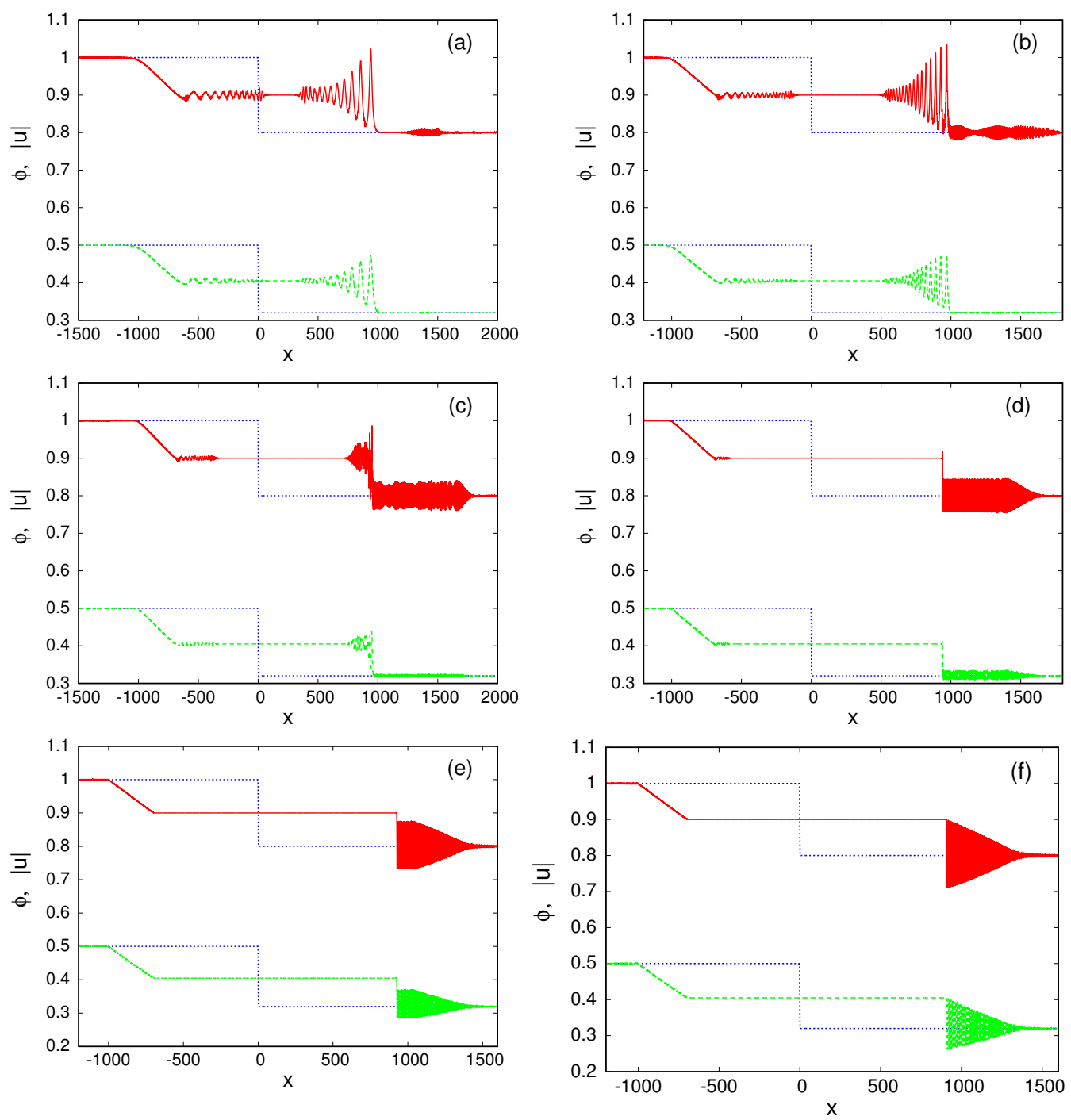

Figure 2. Numerical solutions of nematic Equations (11) and (12) for initial condition (13) with $u_{+}=0.8$ and $u_{-}=1.0$. Red (solid) lines: $|u|$ at $z=1000$; green (dashed) lines: $\phi$ at $z=1000$; blue (dotted) lines: $|u|$ at $z=0$ (upper) and $\phi$ at $z=0$ (lower). (a) PDSW with $v=200$, (b) RDSW with $v=40$, (c) CDSW with $v=10$, (d) TDSW with $v=3.0$, (e) resonant NLS type DSW with $v=1.0$, (f) NLS type DSW with $v=0.5$. Here, $q=2$.

In addition to these six DSW types, when $u_{-}-u_{+}$is large enough as $u_{+} \rightarrow 0$, there is an additional DSW type which is a sub-case of the TDSW regime, the vacuum DSW (VDSW) [37], for which the electric field $u$ of the resonant radiation vanishes at a point $[35,36]$. As the solution for this DSW type has been previously analyzed [36], and it is a sub-case of the TDSW regime, it will not be considered here. In addition, 
the analytical work of this manuscript is based on $u_{-}-u_{+}$being small, which is not valid in the VDSW regime.

Table 1 shows that the transition regimes of the TDSW and RNLS DSWs which evolve the DSW from the KdV-type DSW for high nonlocality to the NLS-type DSW for weak nonlocality exist for very restricted ranges of $v$, so that this transition is rapid. Over most of the range of $v$ the DSW is of KdV-type, one of the PDSW, RDSW, and CDSW types. As noted, the first four DSW regimes also occur for the Kahawara equation and the nematic equations in the low power, high nonlocality limit.

Table 1. Regime boundaries for type classifications of Figure 2 . Here, $u_{-}=1.0, u_{+}=0.8$ and $q=2$.

\begin{tabular}{cc}
\hline PDSW & $88<v$ \\
RDSW & $34<v<88$ \\
CDSW & $4.1<v<34$ \\
TDSW & $1.53<v<4.1$ \\
RNLS DSW & $0.60<v<1.53$ \\
NLS DSW & $0 \leq v<0.60$ \\
\hline
\end{tabular}

The standard method to analyze DSW solutions of nonlinear dispersive wave equations of defocusing NLS-type is to transform the equation into hydrodynamic form using the Madelung transformation [19]

$$
u=\sqrt{\rho} e^{i \psi}, \quad v=\psi_{x}
$$

where the real functions $\rho$ and $\psi$ denote the density and phase of the field $u$, while $v$ is the fluid velocity. Upon substituting, it is found that the nematic Equations (11) and (12) become

$$
\begin{aligned}
\frac{\partial \rho}{\partial z}+\frac{\partial}{\partial x}(\rho v) & =0, \\
\frac{\partial v}{\partial z}+v \frac{\partial v}{\partial x}+2 \frac{\partial \phi}{\partial x}-\frac{\partial}{\partial x}\left(\frac{\rho_{x x}}{4 \rho}-\frac{\rho_{x}^{2}}{8 \rho^{2}}\right) & =0, \\
v \frac{\partial^{2} \phi}{\partial x^{2}}-2 q \phi & =-2 \rho .
\end{aligned}
$$

The nematic equations are characterized by the linear dispersion relation $[34,35]$

$$
\omega=k \bar{v}+\frac{\sqrt{\bar{\rho} k}}{\sqrt{v k^{2}+2 q}}\left[\frac{v k^{2}+2 q}{4 \bar{\rho}} k^{3}+4 k\right]^{1 / 2}
$$

for waves around the mean level $\bar{\rho}$ for $\rho$ and $\bar{v}$ for $v$. In the short wave and high nonlocality limit, $v k^{2} \gg 1$, this dispersion relation can be approximated by

$$
\omega=k \bar{v}+\frac{1}{2} k^{2}+\cdots .
$$

In the opposite local limit with $v$ small, the dispersion relation can be expanded as

$$
\omega=k \bar{v}+k^{2}\left[\frac{1}{4} k^{2}+\frac{2}{q} \bar{\rho}\right]^{1 / 2}-\frac{v \bar{\rho} k^{3}}{2 q^{2}}\left[\frac{1}{4} k^{2}+\frac{2}{q} \bar{\rho}\right]^{-1 / 2}+\cdots
$$

As expected, at the leading-order, $O(1)$, this dispersion relation is the same as that for the NLS equation $[19,50]$. These dispersion relations are needed for the determination of the resonant wavetrain generated by the nematic DSW by which the linear phase velocity is matched to the velocity of the DSW front.

As seen from Figure 2, the solution outside of the DSW and the resonant wavetrain is non-dispersive. On neglecting dispersion, the nematic Equations (15)-(17) become the 
shallow water equations [12], with $\rho$ playing the role of fluid depth and $v$ the (horizontal) fluid velocity. In Riemann invariant form, the dispersionless nematic equations read:

$$
\begin{aligned}
& v+\frac{2 \sqrt{2}}{\sqrt{q}} \sqrt{\rho}=R_{+}=\text {constant } \quad \text { on } \quad C_{+}: \frac{d x}{d z}=V_{+}=v+\frac{\sqrt{2}}{\sqrt{q}} \sqrt{\rho}, \\
& v-\frac{2 \sqrt{2}}{\sqrt{q}} \sqrt{\rho}=R_{-}=\text {constant } \quad \text { on } \quad C_{-}: \frac{d x}{d z}=V_{-}=v-\frac{\sqrt{2}}{\sqrt{q}} \sqrt{\rho} .
\end{aligned}
$$

The initial level behind $u_{-}$is linked to the intermediate shelf by a simple wave on the characteristic $C_{-}$. This simple wave solution has been derived previously [34-36] and is of the form:

$$
|u|=\sqrt{\rho}=\left\{\begin{array}{cc}
u_{-}, & \frac{x}{z}<-\frac{\sqrt{2} u_{-}}{\sqrt{q}} \\
\frac{\sqrt{q}}{3 \sqrt{2}}\left[\frac{2 \sqrt{2} u_{-}}{\sqrt{q}}-\frac{x}{z}\right], & -\frac{\sqrt{2} u_{-}}{\sqrt{q}} \leq \frac{x}{z} \leq \frac{\sqrt{2}}{\sqrt{q}}\left(2 u_{-}-3 \sqrt{\rho_{i}}\right), \\
\sqrt{\rho_{i},} & \frac{\sqrt{2}}{\sqrt{q}}\left(2 u_{-}-3 \sqrt{\rho_{i}}\right)<\frac{x}{z} \leq s_{i}
\end{array}\right.
$$

with $v=2 \sqrt{2}\left(u_{-}-\sqrt{\rho}\right) / \sqrt{q}$, where $s_{i}$ is the velocity of the trailing edge of the DSW which lies on the intermediate level $u_{i}$. This level can be determined by the requirement that the Riemann invariant along the characteristics $C_{-}$, that is, $R_{-}$, is conserved across the nematic DSW [34], giving

$$
u_{i}=\frac{1}{2}\left(u_{-}+u_{+}\right) .
$$

We can see from the above calculations that the phase gradient on the intermediate level $v_{i}$ is then

$$
v_{i}=\frac{2 \sqrt{2}}{\sqrt{q}}\left(u_{-}-\sqrt{\rho_{i}}\right) .
$$

In the small jump limit $\left|u_{-}-u_{+}\right| \ll 1$, the nematic Equations (11) and (12) can be reduced-in the high nonlocality regime $(v \gg 1)$ under consideration-to a KdV equation with fifth-order dispersion $[35,51]$. This will be justified below upon employing a multiscale expansion method.

\subsection{Derivation of the Extended KdV and Kawahara Equations}

We seek solutions of Equations (15)-(17) in the form of the following asymptotic expansions in the formal small parameter $\varepsilon \equiv \sqrt{u_{i}-u_{+}}$(with $0<\varepsilon \ll 1$ ):

$$
\begin{aligned}
|u|^{2} & =\rho=\rho_{+}+\varepsilon^{2} \rho_{1}(\xi, \eta)+\varepsilon^{4} \rho_{2}(\xi, \eta)+\cdots, \\
v & =\varepsilon^{2} V_{1}+\varepsilon^{4} V_{2}+\varepsilon^{6} V_{3}+\cdots \\
\phi & =\frac{\rho_{+}}{q}+\varepsilon^{2} \phi_{1}+\varepsilon^{4} \phi_{2}+\varepsilon^{6} \phi_{3}+\cdots,
\end{aligned}
$$

where $\rho_{+}=u_{+}^{2}$, and the unknown functions $\rho_{j}, V_{j}$, and $\phi_{j}(j=1,2,3, \ldots)$ depend on the stretched variables

$$
\xi=\varepsilon(x-U z), \quad \eta=\varepsilon^{3} z .
$$

Here, $U$ will be treated as an unknown velocity, which will be determined selfconsistently.

Substituting the expansions (26)-(28) into Equations (15)-(17), and using the stretched coordinates (29), we obtain a set of equations at the different orders in $\varepsilon$. In particular, at the leading order, we derive the following linear equations:

$$
O\left(\varepsilon^{2}\right): \quad \rho_{1}-q \phi_{1}=0,
$$


and

$$
O\left(\varepsilon^{3}\right): \quad U V_{1 \xi}-2 \phi_{1 \xi}=0 \text { and } U \rho_{1 \xi}-u_{+}^{2} V_{1 \xi}=0,
$$

where subscripts denote partial derivatives. The compatibility of the above equations suggests that the squared velocity $U^{2}$ is given by $U^{2}=2 u_{+}^{2} / q$. Next, Equations (15)-(17) yield a set of nonlinear equations, namely:

$$
O\left(\varepsilon^{4}\right): \quad 2 \rho_{2}-2 q \phi_{2}+v \phi_{1 \xi \xi}=0,
$$

and

$$
O\left(\varepsilon^{5}\right): \begin{gathered}
-u_{+}^{2} V_{1 \eta}+3 U \rho_{1} V_{1 \xi}-u_{+}^{2} V_{1} V_{1 \xi}+U u_{+}^{2} V_{2 \xi}-6 \rho_{1} \phi_{1 \xi}-2 u_{+}^{2} \phi_{2 \xi}+\frac{1}{4} \rho_{1 \xi \xi \xi}=0 \\
\rho_{1 \eta}+\left(\rho_{1} V_{1}\right)_{\xi}-U \rho_{2 \xi}+u_{+}^{2} V_{2 \xi}=0
\end{gathered} .
$$

The compatibility condition at this order can be found upon eliminating the fields $\rho_{2}$, $V_{2}$, and $\phi_{2}$ upon using Equations (32) and (33) and the definition of the velocity $U$. This yields the following $\mathrm{KdV}$ equation:

$$
\rho_{1 \eta}+\frac{3}{2 u_{+}} \sqrt{\frac{2}{q}} \rho_{1} \rho_{1 \xi}+\left(\frac{1}{q}\right)^{3 / 2} \frac{4 v u_{+}^{2}-q^{2}}{8 \sqrt{2} u_{+}} \rho_{1 \xi \xi \xi}=0 .
$$

To the next order of approximation, we obtain:

$$
O\left(\varepsilon^{6}\right): \quad 2 \rho_{3}-2 q \phi_{3}+v \phi_{2 \xi \xi}=0,
$$

and

$$
\begin{aligned}
O\left(\varepsilon^{7}\right): \quad & -3 u_{+}^{4} \rho_{1} V_{1 \eta}-u_{+}^{6} V_{2 \eta}+3 U u_{+}^{2}\left(\rho_{1}^{2}+u_{+}^{2} \rho_{2}\right) V_{1 \xi}-3 u_{+}^{4} \rho_{1} V_{1} V_{1 \xi} \\
& -u_{+}^{6} V_{2} V_{1 \xi}+3 U u_{+}^{4} \rho_{1} V_{2 \xi}-u_{+}^{6} V_{1} V_{2 \xi}+U u_{+}^{6} V_{3 \xi}-6 u_{+}^{2} \rho_{1}^{2} \phi_{1 \xi} \\
& -6 u_{+}^{4} \rho_{2} \phi_{1 \xi}-6 u_{+}^{4} \rho_{1} \phi_{2 \xi}-2 u_{+}^{6} \phi_{3 \xi}-\frac{1}{2} u_{+}^{2} \rho_{1 \xi} \rho_{1 \xi \xi}+\frac{1}{2} u_{+}^{2} \rho_{1} \rho_{1 \xi \xi \xi} \\
& +\frac{1}{4} u_{+}^{4} \rho_{2 \xi \xi \xi}=0, \quad \text { and } \\
& \rho_{2 \eta}+\left(\rho_{1} V_{2}+\rho_{2} V_{1}\right)_{\xi}-U \rho_{3 \xi}+u_{+}^{2} V_{3 \xi}=0 .
\end{aligned}
$$

It is now possible to follow the procedure used at the previous order and eliminate the fields $\rho_{3}, V_{3}$ and $\phi_{3}$ from Equations (35)-(37). Indeed, solving Equation (35) for $\phi_{3}$, Equation (37) for $\rho_{3 \xi}$ and substituting into Equation (36) eliminates every term with index 3 (recall $U^{2}=2 u_{+}^{2} / q$ ). Furthermore, employing the equations obtained at the previous orders, we can express the fields $\phi_{1,2}$ and $V_{1,2}$ in terms of the amplitudes $\rho_{1}$ and $\rho_{2}$, which yields

$$
\begin{aligned}
& \frac{1}{2 U} \int \rho_{1 \eta \eta} d \xi+\rho_{1 \xi} \int \rho_{1 \eta} d \xi+\frac{5}{2} \rho_{1} \rho_{1 \eta}+\rho_{2 \eta}+3 U \rho_{1}^{2} \rho_{1 \xi}+\frac{3 c}{2}\left(\rho_{1} \rho_{2}\right)_{\xi}+\frac{U v}{2 q} \rho_{1 \xi} \rho_{1 \xi \xi} \\
& -\frac{q-2 U^{2} v}{8 U^{2} q} \rho_{1 \xi \xi \eta}-\frac{q-3 U^{2} v}{4 U q} \rho_{1} \rho_{1 \xi \xi \xi}-\frac{q-2 U^{2} v}{8 U q} \rho_{1 \xi \xi \xi}+\frac{U v^{2}}{8 q^{2}} \rho_{1 \xi \xi \xi \xi \xi}=0 .
\end{aligned}
$$

To this end, we multiply Equation (38) by $\varepsilon^{2}$ and add it to the $\mathrm{KdV}$ equation Equation (34). Then, introducing the combined amplitude function

$$
P=\rho_{1}+\varepsilon^{2} \rho_{2},
$$


we solve for $\rho_{1}=P-\varepsilon \rho_{2}$ and substitute the result into the above Equation (38). We, hence, obtain the nonlinear evolution equation for the field $P(\xi, \eta)$

$$
\begin{gathered}
P_{\eta}+\frac{3}{2 u_{+}} \sqrt{\frac{2}{q}} P P_{\xi}+\left(\frac{1}{q}\right)^{3 / 2} \frac{4 v u_{+}^{2}-q^{2}}{8 \sqrt{2} u_{+}} P_{\xi \xi \xi} \\
+\varepsilon^{2}\left(b_{1} P^{2} P_{\xi}+b_{2} P_{\xi} P_{\xi \xi}+b_{3} P P_{\zeta \xi \xi}+b_{4} P_{\xi \xi \xi \xi \xi}\right)=0 .
\end{gathered}
$$

The coefficients $b_{j}(j=1,2,3,4)$ appearing in Equation (40) are given by

$$
\begin{aligned}
& b_{1}=-\frac{3}{8 u_{+}^{3}} \sqrt{\frac{2}{q}}, \quad b_{2}=-\left(\frac{1}{q}\right)^{3 / 2} \frac{20 v u_{+}^{2}-13 q^{2}}{32 \sqrt{2} u_{+}^{3}} \\
& b_{3}=\left(\frac{1}{q}\right)^{3 / 2} \frac{4 v u_{+}^{2}+q^{2}}{16 \sqrt{2} u_{+}^{3}}, \quad b_{4}=\left(\frac{1}{q}\right)^{5 / 2} \frac{48 v^{2} u_{+}^{4}+8 v q^{2} u_{+}^{2}-q^{4}}{256 \sqrt{2} u_{+}^{3}} .
\end{aligned}
$$

Then, we seek an asymptotic expansion in the optical beam intensity $|u|$ as

$$
|u|=u_{+}+\varepsilon^{2} Q+\cdots
$$

and use the relation $|u|=\sqrt{\rho}$. This asymptotically gives $P=2 u_{+} Q$. The reductive nonlinear Equation (40) can now be written in terms of the field $Q(\xi, \eta)$ as

$$
\begin{gathered}
Q_{\eta}+3 \sqrt{\frac{2}{q}} Q Q_{\xi}+\left(\frac{1}{q}\right)^{3 / 2} \frac{4 v u_{+}^{2}-q^{2}}{8 \sqrt{2} u_{+}} Q_{\xi \xi \xi} \\
+\varepsilon^{2}\left(c_{1} Q^{2} Q_{\xi}+c_{2} Q_{\xi} Q_{\xi \xi}+c_{3} Q Q_{\xi \xi \xi}+c_{4} Q_{\xi \xi \xi \xi \xi}\right)=0 .
\end{gathered}
$$

The coefficients $c_{j}(j=1,2,3,4)$ appearing in Equation (42) are given by

$$
c_{1}=4 u_{+}^{2} b_{1}, \quad c_{2}=2 u_{+} b_{2}, \quad c_{3}=2 u_{+} b_{3}, \quad c_{4}=b_{4} .
$$

Notice that Equation (42) is the so-called extended KdV equation (eKdV), which can model the evolution of steeper waves, with shorter wavelengths, than those governed by the $\mathrm{KdV}$ equation. As such, the $\mathrm{eKdV}$ equation has been used to describe solitary waves in plasmas [52] and shallow water waves [53] in the presence of higher order effects. We note that the coefficient of the third derivative dispersive term changes sign when

$$
v=\frac{q^{2}}{4 u_{+}^{2}}
$$

Hence, in the high nonlocality, low power, limit, such that $v>q^{2} /\left(4 u_{+}^{2}\right)$, the coefficient of the third derivative in eKdV equation is positive, so that its DSW (and solitary wave) solutions have positive polarity, with solitary waves at its leading edge and linear dispersive waves at its trailing edge. On the other hand, in the local limit, $v<q^{2} /\left(4 u_{+}^{2}\right)$, the coefficient of the third derivative is negative and the DSW has negative polarity, with linear dispersive waves at its leading edge and solitary waves at its trailing edge, so that it resembles the standard NLS DSW [37]. The nematic DSW then undergoes a change of form from a KdV-type DSW to an NLS-type DSW as $v$ decreases at the value of the nonlocality parameter given by (43).

At this point, it is useful to make the following remarks. First, in the highly nonlocal limit, the dominant higher-order coefficient is the one of the fifth-order dispersion term, 
namely $c_{4} \propto v^{2}$. Thus, in this limit, the eKdV Equation (40) may be approximated by the Kawahara equation

$$
P_{\eta}+\frac{3}{2 u_{+}} \sqrt{\frac{2}{q}} P P_{\xi}+\left(\frac{1}{q}\right)^{3 / 2} \frac{\left(4 u_{+}^{2} v-q^{2}\right)}{8 \sqrt{2} u_{+}} P_{\xi \xi \xi}+\varepsilon^{2} \frac{3}{16 \sqrt{2}}\left(\frac{1}{q}\right)^{5 / 2} u_{+} v^{2} P_{\xi \xi \xi \xi \xi \xi}=0,
$$

as was done in previous work $[35,36]$.

\section{Nonlocal to Local Nematic DSWs}

The form and details of the DSW solution of the defocusing nematic Equations (11) and (12) will be found as the nonlocality $v$ changes from $O(100)$ to 0 , that is from the nematic having a highly nonlocal response to a local response as the optical power increases. For high nonlocality, the DSW is of KdV type, with the leading edge of the DSW consisting of solitary waves of elevation [34-36]. The reason for this can be seen from the eKdV Equation (42) as for $v>q^{2} /\left(4 u_{+}^{2}\right)$ the coefficient of the third derivative is positive, so that the DSW is of KdV type and has positive polarity. For $0 \leq v<q^{2} /\left(4 u_{+}^{2}\right)$, the sign of the third derivative is negative, and the DSW has negative polarity, with solitary waves at the trailing edge and linear dispersive waves at the leading edge, as for the NLS DSW [37]. A nematic DSW of NLS type is illustrated in Figure 2e for $v=0.5$. Indeed, for $v=0$, the nematic Equations (11) and (12) reduce to the standard NLS equation on substituting for $\phi$ from (12) into (11). The DSW solution of the NLS equation is well known [37] as the NLS equation is integrable and so the solution is completely determined. Hence, the solution for this regime will not be considered here.

The existence regions for the various nematic DSW types as the nonlocality parameter $v$ varies (optical power varies), found from full numerical solutions of the nematic Equations (11) and (12) are shown in Figure 3 as the initial level ahead $u_{+}$varies. The range $0.3 \leq u_{+} \leq 0.9$ was chosen as this encompassed all six of the DSW types studied here. In addition, most of the theoretical expressions for the boundaries between these regions and the solutions within each region were based on $u_{-}-u_{+}$small, for example, the boundary (43), which is based on the eKdV Equation (42). It can be seen that, over most of the $\left(u_{+}, v\right)$ domain, the nematic DSW is of CDSW or TDSW type, so that it is typically unstable. In addition, it is deduced that the nematic DSW is of NLS-type only for small values of the nonlocality parameter $v$ of 2 and below. The nematic DSW is then nonlocal, except for high enough optical powers for which $v$ is small. The nonlocality parameter $v$ is given by (9). For the nematic liquid crystal 4-(trans-4-n-hexylcyclohexyl)-isothiocyanato-benzene (6CHBT), the parameter values are $K \sim 10^{-11} N, n_{\|}=1.6335$ and $n_{\perp}=1.4967$ [54]. Let us take the pre-tilt angle $\theta_{0}$ to be $\pi / 4$ so that the nematic response is maximized [2]. A typical beam wavelength is $1064 \mathrm{~nm}$, and a typical half width $W_{b}$ is $1.5 \mu \mathrm{m}$ [54]. With these parameter values, it is found that $v=2$ when the beam power $P_{b}$ is $288 \mathrm{~mW}$, far in excess of typical beam powers of a few milliWatts to a few tens of milliWatts [2,54]. Such a large optical power can result in the nematic medium being heated enough so that its temperature goes above the critical temperature, $43^{\circ} \mathrm{C}$ for $6 \mathrm{CHBT}$ [54], so that it undergoes a phase change out of the nematic state. In this regard, it should be noted that experimental nematic cells are small, of the order of $1 \mathrm{~mm}$ in the down cell propagation direction of the beam and $100 \mu \mathrm{m} \times 10 \mathrm{~mm}$ in cross-section. The thin cross-section is the direction in which the pre-tilting electric field is applied, which results in a stable and uniform molecular pre-tilt. We then deduce that, for experimental beam powers, the nematic bore will be in the nonlocal response regime with $v$ large, which is low optical power. 

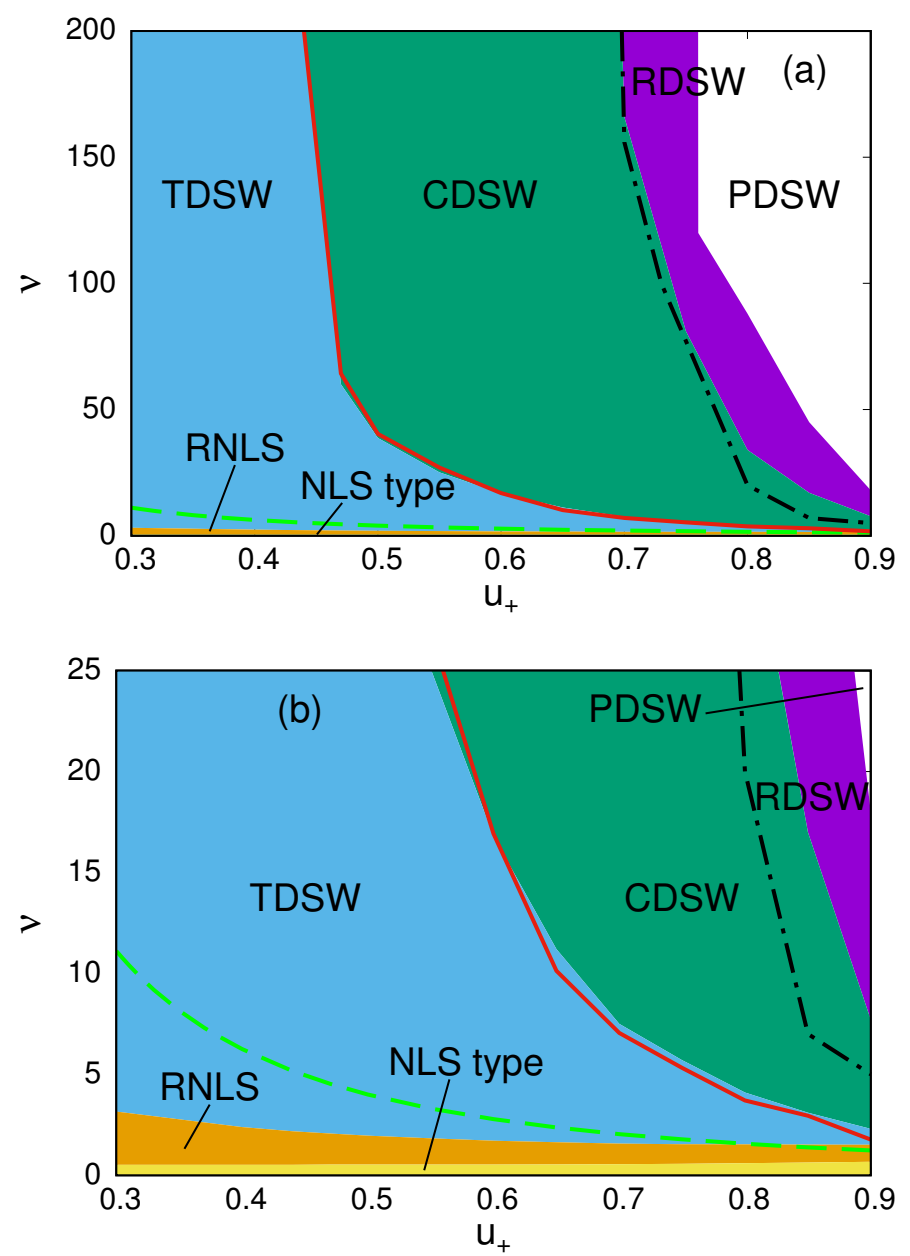

Figure 3. Numerical existence regions for nematic DSW types in terms of the nonlocality parameter $v$ as the initial level $u_{+}$varies. (a) Full existence regions from highly nonlocal $v=200$ to local $v=0$, (b) Detail of (a) for the transition to an NLS-type DSW. Theoretical boundary (72) between RDSW and CDSW regimes: black (dot-dash) line; theoretical boundary $U_{s}=c_{g}$, (95), between CDSW and TDSW regimes: red (solid) line; theoretical boundary (43) between KdV-type and NLS-type DSW: green (dashed) line. Here, $u_{-}=1.0$ and $q=2$.

\section{PDSW and RDSW Regimes}

Typical PDSW and RDSW solutions are illustrated in Figure 2a,b. In the PDSW regime, the DSW is not in resonance with linear diffractive radiation, so that the DSW is of KdV type, as seen in the boundary (43), and is a perturbed KdV-type DSW. In the RDSW regime, the DSW is in resonance with diffractive radiation, so that it consists of a KdV-type DSW with resonant radiation propagating ahead of it. This resonant radiation is not large enough, however, as to destroy the KdV-type DSW structure, as in the CDSW regime; see Figure 2c. As the DSW in the PDSW and RDSW regimes are perturbed KdV DSWs [36], the solutions in these two regimes can be found using the perturbed KdV DSW solution of Reference [47]. In this previous work, the general eKdV equation, a particular case of which is (42), was asymptotically transformed to the KdV equation, in which the known DSW solution [19,23] was then used to find the asymptotic DSW solution of the original eKdV equation. This asymptotic DSW solution can be used here based on the eKdV equation reduction (42) of the full nematic equations in the limit $u_{-}-u_{+}$small. The work [47] then gives the following PDSW and RDSW nematic DSW solutions. The amplitude of the DSW is

$$
\begin{aligned}
& a=2 m\left(u_{i}-u_{+}\right)+\frac{1}{3}\left(u_{i}-u_{+}\right)^{2}\left\{m(1-m) C_{1}+m(m-2) C_{2}\right. \\
& \left.\quad+m C_{3}+2 m(8-3 m) C_{4}\right\}
\end{aligned}
$$


its wavenumber is

$$
\begin{aligned}
& k=\frac{\pi \sqrt{2\left\{u_{i}-u_{+}\right\}}}{K(m) \sqrt{u_{+}} \sqrt{\frac{v}{q}-\frac{q}{4 u_{+}^{2}}}}\left\{1+\frac{\left(u_{i}-u_{+}\right)}{12} C_{1}+\frac{\left(u_{i}-u_{+}\right)\left(4 m^{2}-8 m+3\right)}{12} C_{3}\right. \\
& \left.-\frac{\left(u_{i}-u_{+}\right)\left(8 m^{2}-14 m+11\right)}{3} C_{4}\right\},
\end{aligned}
$$

and its mean level is

$$
\begin{aligned}
& |\bar{u}|=2 u_{+}-u_{i}+\left(u_{i}-u_{+}\right)\left\{m+\frac{2 E(m)}{K(m)}\right\} \\
& -\left(u_{i}-u_{+}\right)^{2} C_{1}\left\{\frac{3 m^{2} K(m)+4 m E(m)-5 m K(m)-2 E(m)+2 K(m)}{18 K(m)}\right\} \\
& -\left(u_{i}-u_{+}\right)^{2} C_{3}\left\{\frac{\left.\left(2-m-m^{2}\right) K^{2}(m)+(4 m-10) E(m) K(m)+8 E^{2}(m)\right)}{6 K^{2}(m)}\right\} \\
& +2\left(u_{i}-u_{+}\right)^{2} C_{4}\left\{\frac{\left(m^{2}-7 m+6\right) K^{2}(m)+2(6 m-11) E(m) K(m)+16 E^{2}(m)}{3 K^{2}(m)}\right\} .
\end{aligned}
$$

Here, $K(m)$ and $E(m)$ are complete elliptic integrals of the first and second kinds of modulus squared $m$, respectively. The modulus squared $m$ is a parameter in these amplitude, wavelength, and mean height expressions. It is determined in terms of the simple wave (similarity) variable $x / z$ by

$$
\begin{aligned}
& \frac{x}{z}=\frac{\sqrt{2}}{\sqrt{q}} u_{+}+\frac{\sqrt{2}\left(u_{i}-u_{+}\right)}{\sqrt{q}}\left\{1+m-\frac{2 m(1-m) K(m)}{E(m)+(m-1) K(m)}\right\} \\
& +\frac{\left(u_{i}-u_{+}\right)^{2}}{3 \sqrt{2 q}} C_{1}\left\{1+m-\frac{2 m(1-m) K(m)}{E(m)+(m-1) K(m)}\right\} \\
& +\frac{\left(u_{i}-u_{+}\right)^{2}}{3 \sqrt{2 q}} C_{3}\left\{2 m-1-m^{2}-\left(1+m-\frac{2 m(1-m) K(m)}{E(m)+(m-1) K(m)}\right)\right. \\
& \left.-\frac{4 m(1-m)(2 E(m)+(m-1) K(m))}{E(m)+(m-1) K(m)}\right\}+\frac{4\left(u_{i}-u_{+}\right)^{2}}{3 \sqrt{2 q}} C_{4} \\
& \times\left\{-1-m+\frac{2 m(1-m) K(m)}{E(m)+(m-1) K(m)}+\left(1+m-\frac{2 m(1-m) K(m)}{E(m)+(m-1) K(m)}\right)^{2}\right. \\
& \left.-2\left(2 m-m^{2}-1\right)+4 \frac{m(1-m)[(m-1) K(m)+2 E(m)]}{E(m)+(m-1) K(m)}\right\} .
\end{aligned}
$$

This expression for $x / z$ derives from the characteristic of the $\mathrm{KdV}$ modulation equations on which the simple wave DSW solution occurs $[23,24,47]$. The coefficients $C_{j}(j=$ $1,2,3,4)$ in the above solutions are connected to $c_{j}(j=1,2,3,4)$ through the relations

$$
C_{1}=\sqrt{2 q} c_{1}, \quad C_{2}=\frac{8 \sqrt{2} q^{3 / 2} u_{+}}{\left(4 u_{+}^{2} v-q^{2}\right)} c_{2}, \quad C_{3}=\frac{8 \sqrt{2} q^{3 / 2} u_{+}}{\left(4 u_{+}^{2} v-q^{2}\right)} c_{3}, \quad c_{4}=\frac{64 \sqrt{2} q^{5 / 2} u_{+}^{2}}{\left(4 u_{+}^{2} v-q^{2}\right)^{2}} c_{4} .
$$

At the leading, solitary wave edge of the DSW $m \rightarrow 1$ and at the trailing, harmonic wave edge of the DSW $m \rightarrow 0$. It can then be found from the characteristics (48) that the DSW lies in the range

$$
\begin{aligned}
s_{i}=\sqrt{\frac{2}{q}}\left\{4 u_{+}-3 u_{i}\right. & \left.-\left(u_{i}-u_{+}\right)^{2}\left(\frac{1}{2} C_{1}+C_{3}-\frac{64}{3} C_{4}\right)\right\} \\
& \leq \frac{x}{z} \leq \sqrt{\frac{2}{q}}\left\{2 u_{i}-u_{+}+\frac{1}{3}\left(u_{i}-u_{+}\right)^{2}\left(C_{1}-C_{3}+4 C_{4}\right)\right\}=s_{+},
\end{aligned}
$$

where $s_{i}$ and $s_{+}$are the harmonic and solitary wave edge velocities of the DSW, respectively.

Comparisons between the lead solitary wave amplitude $a_{+}$as given by the asymptotic DSW solution (45), with $m=1$, and numerical solutions are given in Figure 4 as the nonlocality parameter $v$ varies. The existence regions for the PDSW and RDSW types depend on both $u_{+}$and $v$, as shown in Figure 3, so that the comparison curves for each $u_{+}$ were stopped at the boundary between the RDSW and CDSW regimes. Figure 4 shows the lead solitary wave amplitude for the full eKdV Equation (42) and the Kawahara equation, 
which is (42) with $c_{1}=c_{2}=c_{3}=0$. Previous work on the nematic bore $[35,36]$ was based on the Kawahara equation, that is only the higher order fifth derivative was included in the asymptotic eKdV equation, so the lead wave amplitude based on this equation is given in the figure to determine the effect of the extra higher order terms in the full eKdV Equation (42). A key observation is that the height of the lead wave of the DSW depends very weakly on the strength of the nonlocality, with little variation even down to $v=O(10)$ from the high nonlocality amplitudes with $v=O(100)$. In some sense, the nematic DSW is then nonlocal down to small values of $v$ in the PDSW and RDSW regimes, which was also deduced above from Figure 3. It can also be seen that the inclusion of the extra higher order terms in the eKdV Equation (42) over the Kawahara equation improves the agreement with numerical solutions on the whole, especially as the nonlocality parameter $v$ decreases, but the effect of these extra terms is small, with the Kawahara equation giving good agreement over the whole range of $v$ and for all values of $u_{+}$, except near the RDSW/CDSW borderline at $u_{+}=0.7$. This is expected as the weak dependence on the nonlocality parameter $v$ means that the DSW is nonlocal, so that $v$ can be taken as large. The dominant higher order term in the eKdV equation is $\varepsilon^{2} c_{4} Q_{\xi \xi \xi \xi \xi}$ in this limit, as noted in (44). As $u_{+}=0.7$ is approached the resonant radiation shed by the DSW is of relatively large amplitude as in this limit the RDSW/CDSW boundary is approached. This results in oscillations in the lead wave amplitude as the resonant radiation moves through the DSW and is shed. In these cases, the numerical amplitude shown in Figure 4 was calculated as an average in $z$ over the last few amplitude oscillations in the numerical solution. It is noted that except for $u_{+}=0.75$ and 0.7 the amplitude grows as $v$ decreases. This change in behavior is due to the DSW changing form as it transitions from the RDSW to the CDSW regime, for which the resonant radiation has a major effect on the DSW with its amplitude decreasing markedly due to the large amount of mass being shed as resonant radiation [36].

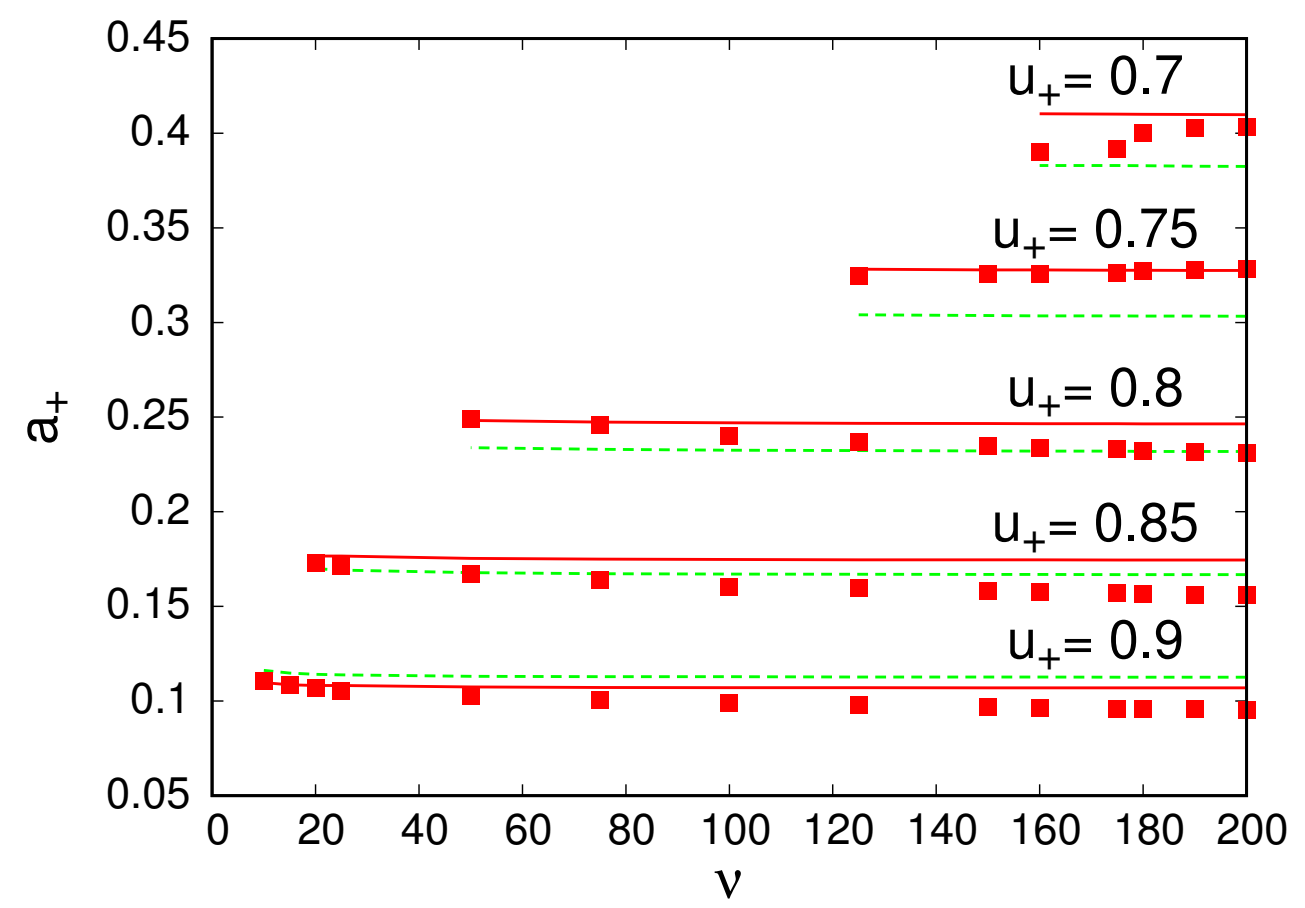

Figure 4. Comparison between lead wave amplitude $a_{+}$of PDSW and RSDW with numerical solutions of the nematic Equations (11) and (12) with the initial condition (13). Numerical amplitude: red squares; amplitude (45) with $m=1$ given by undular bore solution of eKdV Equation (42): red (solid) line; amplitude given by undular bore solution of Kawahara equation (Equation (42) with $c_{1}=c_{2}=c_{3}=0$ ): green (dashed) line. Here, $u_{-}=1.0$ and $q=2$. 
The full linear dispersion relation (18) for the nematic Equations (11) and (12) is

$$
\omega=k \bar{v}+\frac{\sqrt{\bar{\rho}} k}{\sqrt{v k^{2}+2 q}}\left[\frac{v k^{2}+2 q}{4 \bar{\rho}} k^{2}+4\right]^{1 / 2}+\frac{2 \bar{\rho}}{q}
$$

when the mean $2 \bar{\rho} / q$ is added. This mean term arises on integrating $v=\psi_{x}$ to obtain the dispersion relation for $\psi$ from that for $v$ [35]. In the limit $v k^{2}$ large, this dispersion relation becomes (19)

$$
\omega=k \bar{v}+\frac{1}{2} k^{2}+\frac{2 \bar{\rho}}{q}+\ldots
$$

again with the carrier wave phase shift $2 \bar{\rho} / q$ added to obtain the dispersion relation for $\psi$. In the RDSW regime, the resonant wavetrain has low amplitude; see Figure $2 \mathrm{~b}$. In addition, this wavetrain has high frequency relative to the DSW; again, see Figure $2 \mathrm{~b}$. So, the appropriate dispersion relation for the resonant wavetrain is (52). In the RDSW regime, the resonance condition for the resonant wavetrain ahead of the DSW has been set by matching the phase velocity of the resonant wavetrain to the velocity of the lead wave of the DSW [34-36], giving

$$
s_{+}=c_{r}, \quad c=\bar{v}+\frac{1}{2} k+\frac{2 \bar{\rho}}{q k}
$$

so that

$$
k_{r}=s_{+}+\left[s_{+}^{2}-\frac{4}{q} u_{+}^{2}\right]^{1 / 2}
$$

on setting $\bar{\rho}=\sqrt{u_{+}}$and $\bar{v}_{+}=0$ as the resonant wavetrain propagates on the level ahead. This gives the wavenumber of the resonant wavetrain based on this criterion. The resonant wavetrain then exists if $s_{+} \geq 2 u_{+} / \sqrt{q}$, so that the borderline between the PDSW and RDSW regimes is $s_{+}=2 u_{+} / \sqrt{q}$. Previous work $[35,36]$ has shown that this theoretical borderline is in excellent agreement with numerical solutions in the high nonlocality limit $v$ large. For fixed $u_{+}$, as $v$ decreases, a PDSW changes to an RDSW, then to a CDSW; see Figure 3. For instance, for $u_{+}=0.8$, the DSW changes from PDSW to RDSW at $v=88$, then to CDSW at $v=34$. However, the resonance condition (53), or (54), gives that the DSW changes from PDSW to RDSW at $v=3$, which is the TDSW regime according to Table 1. This resonance condition is based on the limit $v k^{2} \gg 1$, but, even if the full dispersion relation (51) were used for the resonance condition (53), the predicted PDSW/RDSW borderline is $v=2.38$, which is still far from the numerical value and close to that for $v k^{2} \gg 1$. The resonance condition (53) is based on resonance between the lead wave of the DSW and diffractive radiation. However, as pointed out previously [36], a DSW is modulated periodic wave so that all waves of the DSW can resonant with diffractive radiation, not just the lead wave, as seen in Figure 5 for a PDSW. Internal resonance will be discussed in detail in Section 5. The phase velocity of a component wave of the DSW is (72). Equating this bore component phase velocity with the nematic diffractive radiation phase velocity determined from the dispersion relation (18) determines the internal resonance. However, even using this internal resonance does not result in a borderline between the PDSW and RDSW regimes in any reasonable accord with numerical solutions; see Figure 3. A resonant wavetrain will then exist if the internally resonant waves can propagate out of the DSW, that is their group velocity is greater than the velocity of the lead solitary wave of the DSW. However, even this condition does not give the correct boundary between the PDSW and RDSW regimes as the nonlocality parameter $v$ decreases. The issue of internal resonance and its relation to the existence of the PDSW and RDSW regimes merits further study. In this regard, the recent work [55] on the interaction of linear wavepackets and DSWs could be relevant. 


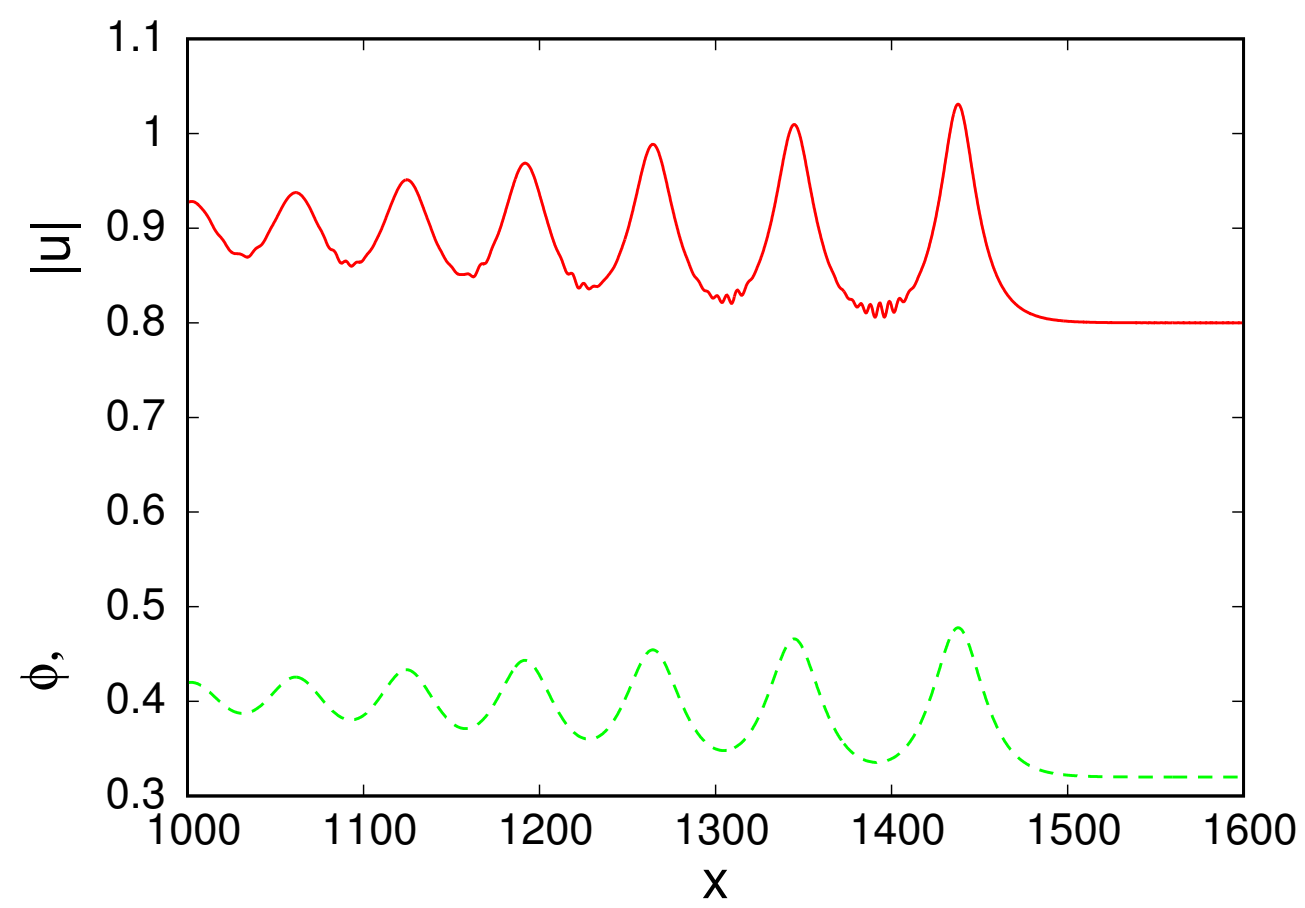

Figure 5. Numerical solution of nematic Equations (11) and (12) in PDSW regime at $z=1500$ for initial condition (13) with $u_{+}=0.8$ and $u_{-}=1.0$. Red (solid) line: $|u|$; green (dashed) line: $\phi$. Here, $v=200$ and $q=2$.

\section{CDSW Regime}

For fixed a nonlocality parameter $v$, as $u_{+}$decreases, the jump height $u_{-}-u_{+}$increases, the nematic DSW changes from RDSW to CDSW form; see Figure $3 b$. The reason for this is that, as the amplitude of the resonant wavetrain grows, it takes more conserved quantities from the DSW, so that its amplitude is reduced. In addition, the DSW becomes unstable in the CDSW regime, as for the Kahawara equation DSW [26], noting that, in the small jump height limit, the nematic equations reduce to the eKdV Equation (42), which becomes the Kahawara Equation (44) in the limit of large nonlocality $v$. Figure 2c shows a typical nematic DSW in the CDSW regime. It can be seen that the DSW has changed from a modulated wavetrain with a monotonically decreasing amplitude from front to rear to a disordered wavetrain with an essentially uniform amplitude on average, except at its rear. This structure is in agreement with unstable DSW structure for the focusing NLS equation [56]. The unstable DSW can then be approximated by a train of equal amplitude solitary waves, which has been found to give good results for DSW solutions [57], particularly unstable DSWs, and for the particular case of the nematic CDSW in the high nonlocality, low optical power, regime [36]. The amplitude of the solitary waves of the CDSW is determined from mass and energy conservation equations for the underlying nonlinear dispersive wave equation [57].

If we set

$$
B_{2}=3 \sqrt{\frac{2}{q}}, \quad B_{3}=\frac{4 v u_{+}^{2}-q^{2}}{8 \sqrt{2} q^{3 / 2} u_{+}}
$$

for simplicity, the eKdV Equation (42) has the mass conservation equation

$$
\begin{aligned}
& \frac{\partial}{\partial \eta} Q+\frac{\partial}{\partial \xi}\left[\frac{1}{2} B_{2} Q^{2}+B_{3} Q_{\xi \xi}+\frac{1}{3} \varepsilon^{2} c_{1} Q^{3}+\frac{1}{2} \varepsilon^{2}\left(c_{2}-c_{3}\right) Q_{\xi}^{2}+\varepsilon^{2} c_{3} Q Q_{\xi \xi}\right. \\
& \left.+\varepsilon^{2} c_{4} Q_{\xi \xi \xi \xi}\right]=0 .
\end{aligned}
$$


The derivation of the energy conservation for the eKdV Equation (42) is not as straightforward. Multiplying the eKdV equation by $Q$ and integrating by parts gives

$$
\begin{aligned}
& \frac{\partial}{\partial \eta} \frac{1}{2} Q^{2}+\frac{\partial}{\partial \xi}\left[\frac{1}{3} B_{2} Q^{3}+B_{3} Q Q_{\xi \xi}-\frac{1}{2} B_{3} Q_{\xi}^{2}+\frac{1}{4} \varepsilon^{2} c_{1} Q^{4}+\frac{1}{2} \varepsilon^{2} c_{2} Q^{2} Q_{\xi \xi}\right. \\
& \left.+\varepsilon^{2} c_{4} Q Q_{\xi \xi \xi \xi}-\varepsilon^{2} c_{4} Q_{\xi} Q_{\xi \xi \xi}+\frac{1}{2} \varepsilon^{2} c_{4} Q_{\xi \xi}^{2}\right]+\varepsilon^{2}\left(c_{3}-\frac{1}{2} c_{2}\right) Q^{2} Q_{\xi \zeta \xi}=0 .
\end{aligned}
$$

The final term on the right hand side of this equation cannot be expressed as a perfect derivative. However, it can be approximately expressed in this form on noting that $\varepsilon$ is small, so that, at first order, the eKdV Equation (42) is the KdV equation

$$
\frac{\partial Q}{\partial \eta}+B_{2} Q \frac{\partial Q}{\partial \xi}+B_{3} \frac{\partial^{3} Q}{\partial \xi^{3}}=0
$$

We then have at leading order that

$$
\frac{\partial}{\partial \eta} Q^{3}=-3 Q^{2}\left(B_{2} Q Q_{\xi}+B_{3} Q_{\xi \xi \xi}\right)=-\frac{\partial}{\partial \xi} \frac{3}{4} B_{2} Q^{4}-3 B_{3} Q^{2} Q_{\xi \xi \xi} .
$$

This expression may now be used to eliminate the term in $Q Q_{\xi \xi \xi}$ in Equation (57) to give the final energy conservation equation

$$
\begin{aligned}
& \frac{\partial}{\partial \eta}\left[\frac{1}{2} Q^{2}-\varepsilon^{2} \frac{c_{3}-\frac{1}{2} c_{2}}{3 B_{3}} Q^{3}\right]+\frac{\partial}{\partial \xi}\left[\frac{1}{3} B_{2} Q^{3}+B_{3} Q Q_{\xi \xi}-\frac{1}{2} B_{3} Q_{\xi}^{2}+\frac{1}{4} \varepsilon^{2} c_{1} Q^{4}\right. \\
& +\frac{1}{2} \varepsilon^{2} c_{2} Q^{2} Q_{\xi \xi}+\varepsilon^{2} c_{4} Q Q_{\xi \xi \xi \xi}-\varepsilon^{2} c_{4} Q_{\xi} Q_{\xi \xi \xi}+\frac{1}{2} \varepsilon^{2} c_{4} Q_{\xi \xi}^{2} \\
& \left.-\varepsilon^{2} \frac{B_{2}}{4 B_{3}}\left(c_{3}-\frac{1}{2} c_{2}\right) Q^{4}\right]=0,
\end{aligned}
$$

which is accurate to $O\left(\varepsilon^{2}\right)$.

To obtain an approximation to the nematic CDSW, let us assume that, at position $\eta$, the CDSW consists of $N$ equal solitary waves of amplitude $\tilde{a}_{S}$ and width $\tilde{w}_{S}$ [57], where we shall use tildes to denote scaled variables in the moving and stretched coordinates $(\xi, \eta)$. It is also assumed that the CDSW sheds a uniform downstream resonant wavetrain of (scaled) amplitude $\tilde{a}_{r}$. Then, as $\xi \rightarrow-\infty, Q \rightarrow 1$ and as $\xi \rightarrow \infty, Q \rightarrow \tilde{a}_{r} \cos \left(\tilde{k}_{r} \xi-\tilde{\omega}_{r} \eta\right)$, since $|u|=u_{+}+\varepsilon^{2} Q$ with $\varepsilon^{2}=u_{i}-u_{+}$. As the CDSW is approximated by a train of solitary waves, the solitary wave solution of the eKdV Equation (42) is also needed. While there is no known exact solitary wave solution of this equation, there is an asymptotic solution for $\varepsilon \ll 1$ [58]. To use this solution, the eKdV Equation (42) needs to be rescaled to conform with the eKdV scaling of Reference [58]. Performing this, we find that the asymptotic solitary wave solution of the eKdV Equation (42) is

$$
Q=\gamma_{1} \operatorname{sech}^{2} \frac{\xi-V_{s} \eta}{W}+\gamma_{2} \operatorname{sech}^{4} \frac{\xi-V_{s} \eta}{W}, \quad V_{s}=\frac{1}{3} B_{2} A\left(1+2 \varepsilon^{2} C_{4} A\right),
$$

where

$$
W=\frac{\sqrt{12 B_{3}}}{\sqrt{B_{2} A}}, \quad \gamma_{1}=A+\varepsilon^{2} C_{6} A^{2}, \quad \gamma_{2}=\varepsilon^{2} C_{7} A^{2} .
$$

The rescaled coefficients $c_{i}, i=1, \ldots, 4$, of the eKdV Equation (42), denoted by $C_{i}$, $i=1, \ldots, 4$, are given by

$$
\begin{array}{cl}
C_{1}=\frac{6}{B_{2}} c_{1}, \quad C_{2}=\frac{1}{B_{3}} c_{2}, & C_{3}=\frac{1}{B_{3}} c_{3}, \quad C_{4}=\frac{B_{2}}{6 B_{3}^{2}} c_{4}, \\
C_{6}=\frac{2}{3} C_{3}-\frac{1}{6} C_{1}+\frac{1}{6} C_{2}-5 C_{4}, & C_{7}=\frac{15}{2} C_{4}-\frac{1}{2} C_{3}+\frac{1}{12} C_{1}-\frac{1}{4} C_{2} .
\end{array}
$$

It is noted that these scaled $C_{i}, i=1, \ldots, 4$, are the same as the $C_{i}(49)$ used for the perturbed DSW solution (45)-(48) due to the same rescaling from the eKdV Equation (42) being used for this solution. 
The mass and energy conservation Equations (56) and (60) can now be used to determine the amplitude of the solitary waves in the nematic CDSW in the equal amplitude approximation. The mass and energy conservation equations are integrated in $\xi$ from $-\infty$ to $\infty$. The integral of the mass and energy densities are approximated by $N$ times these for a single solitary wave (61). The flux terms are calculated using the boundary conditions stated above, $Q=1$ at $\xi=-\infty$ and $Q=\tilde{a}_{r} \cos \left(\tilde{k}_{r} \xi-\tilde{\omega}_{r} \eta\right)$ at $\xi=\infty$. The resonant radiation flux at $\xi=\infty$ is calculated by averaging the periodic radiation over a period [36]. In this manner, integrating the mass conservation equation gives

$$
N\left[2 \gamma_{1}+\frac{4}{3} \gamma_{2}\right] W=\left\{\frac{1}{2} B_{2}+\frac{1}{3} \varepsilon^{2} c_{1}-\left[\frac{1}{4} B_{2}+\frac{1}{4} \varepsilon^{2}\left(c_{2}-3 c_{3}\right) \tilde{k}_{r}^{2}\right] \tilde{a}_{r}^{2}\right\} \eta
$$

and integrating the energy conservation equation gives

$$
\begin{aligned}
& N\left[\frac{2}{3} \gamma_{1}^{2}+\frac{16}{15} \gamma_{1} \gamma_{2}-\varepsilon^{2} \frac{16}{45} \frac{c_{3}-\frac{1}{2} c_{2}}{B_{3}} \gamma_{1}^{3}\right] W=\left\{\frac{1}{3} B_{2}+\frac{1}{4} \varepsilon^{2} c_{1}\right. \\
& -\varepsilon^{2} \frac{B_{2}}{4 B_{3}}\left(c_{3}-\frac{1}{2} c_{2}\right)-\frac{1}{4}\left[-3 B_{3} \tilde{k}_{r}^{2} \tilde{a}_{r}^{2}+\frac{3}{8} \varepsilon^{2} c_{1} \tilde{a}_{r}^{4}+5 \varepsilon^{2} c_{4} \tilde{k}_{r}^{4} \tilde{a}_{r}^{2}\right. \\
& \left.\left.-\varepsilon^{2} \frac{3 B_{2}}{8 B_{3}}\left(c_{3}-\frac{1}{2} c_{2}\right) \tilde{a}_{r}^{4}\right]\right\} \eta \\
& \sim\left\{\frac{1}{3} B_{2}+\frac{1}{4} \varepsilon^{2} c_{1}-\varepsilon^{2} \frac{B_{2}}{4 B_{3}}\left(c_{3}-\frac{1}{2} c_{2}\right)-\frac{1}{4} \tilde{c}_{g} \tilde{a}_{r}^{2}\right\} \eta
\end{aligned}
$$

since $\tilde{a}_{r}$ is small. In addition, this neglect of quartic terms in $\tilde{a}_{r}$ is consistent with the radiation being determined by a linear WKB analysis [35]. Here, $\tilde{c}_{g}$ is the scaled group velocity of the resonant radiation based on (29). Dividing the mass and energy Equations (64) and (65) gives an equation for $A$

$$
\frac{\gamma_{1}^{2}+\frac{8}{5} \gamma_{1} \gamma_{2}-\varepsilon^{2} \frac{8}{15} \frac{c_{3}-\frac{1}{2} c_{2}}{B_{3}} \gamma_{1}^{3}}{\gamma_{1}+\frac{2}{3} \gamma_{2}}=4 \frac{B_{2}+\frac{3}{4} \varepsilon^{2} c_{1}-\varepsilon^{2} \frac{3 B_{2}}{4 B_{3}}\left(c_{3}-\frac{1}{2} c_{2}\right)-\frac{3}{4} \tilde{c}_{r} \tilde{a}_{r}^{2}}{2 B_{2}+\frac{4}{3} \varepsilon^{2} c_{1}-\left[B_{2}+\varepsilon^{2}\left(c_{2}-3 c_{3}\right) \tilde{k}_{r}^{2}\right] \tilde{a}_{r}^{2}}
$$

in terms of $\tilde{a}_{r}$. Once $A$ is determined, the unscaled amplitude $a_{s}$ of the solitary waves of the CDSW is given by

$$
a_{S}=\varepsilon^{2}\left[A+\varepsilon^{2}\left(C_{6}+C_{7}\right) A^{2}\right]=\left(u_{i}-u_{+}\right)\left[A+\left(u_{i}-u_{+}\right)\left(C_{6}+C_{7}\right) A^{2}\right],
$$

on using the solitary wave solution (61). Transforming back from the scaled eKdV variables to the original variables, the relation (66) becomes

$$
\begin{aligned}
& \frac{\gamma_{1}^{2}+\frac{8}{5} \gamma_{1} \gamma_{2}-\frac{8}{15}\left(u_{i}-u_{+}\right) \frac{c_{3}-\frac{1}{2} c_{2}}{B_{3}} \gamma_{1}^{3}}{\gamma_{1}+\frac{2}{3} \gamma_{2}} \\
& =4 \frac{B_{2}+\frac{3}{4}\left(u_{i}-u_{+}\right) c_{1}-\frac{3 B_{2}}{4 B_{3}}\left(u_{i}-u_{+}\right)\left(c_{3}-\frac{1}{2} c_{2}\right)-\frac{3}{4} \frac{\left(c_{g}-\sqrt{\frac{2}{9}} u_{+}\right) a_{r}^{2}}{\left(u_{i}-u_{+}\right)^{3}}}{2 B_{2}+\frac{4}{3}\left(u_{i}-u_{+}\right) c_{1}-\left[B_{2}+\left(c_{2}-3 c_{3}\right) k_{r}^{2}\right] \frac{a_{r}^{2}}{\left(u_{i}-u_{+}\right)^{2}}}
\end{aligned}
$$


on using the scalings (63) for the eKdV equation. Substituting for the higher order solitary wave coefficients $\gamma_{1}$ and $\gamma_{2}$ given by (62) gives the final equation determining the amplitude of the CDSW solitary waves as

$$
\begin{aligned}
& A \frac{1+2\left(u_{i}-u_{+}\right)\left(C_{6}+\frac{4}{5} C_{7}\right) A-\frac{8}{15}\left(u_{i}-u_{+}\right)\left(C_{3}-\frac{1}{2} C_{2}\right) A}{1+\left(u_{i}-u_{+}\right)\left(C_{6}+\frac{2}{3} C_{7}\right) A} \\
& =4 \frac{B_{2}+\frac{1}{8}\left(u_{i}-u_{+}\right) B_{2}\left(C_{1}+3 C_{2}-6 C_{3}\right)-\frac{3}{4} \frac{\left(c_{g}-\sqrt{\frac{2}{9}} u_{+}\right) a_{r}^{2}}{\left(u_{i}-u_{+}\right)^{3}}}{2 B_{2}+\frac{2}{9}\left(u_{i}-u_{+}\right) B_{2} C_{1}-\left[B_{2}+\left(c_{2}-3 c_{3}\right) k_{r}^{2}\right] \frac{a_{r}^{2}}{\left(u_{i}-u_{+}\right)^{2}}} .
\end{aligned}
$$

The final quantity to determine is the amplitude of $a_{r}$ of the resonant radiation, which is related to the scaled amplitude $\tilde{a}_{r}$ by $a_{r}=\varepsilon^{2} \tilde{a}_{r}=\left(u_{i}-u_{+}\right) \tilde{a}_{r}$. This resonant radiation was determined as a WKB solution of the nematic Equations (11) and (12) by linearizing about the mean level $u_{+}$of the resonant radiation [35]. This WKB solution gives the amplitude of the resonant radiation as

$$
a_{r}=\frac{1}{2} \frac{u_{-}-u_{+}}{1+\frac{2 u_{+} k_{r} a_{s}}{q s_{+}\left(k_{r}-s_{+}\right)^{2}}} .
$$

Here, $s_{+}$is the unscaled velocity of the CDSW, which is [36]

$$
s_{+}=\sqrt{\frac{2}{q}} u_{+}+\frac{1}{3} B_{2} a_{s}\left(1+2 C_{4} a_{s}\right) .
$$

The resonant radiation wavenumber $k_{r}$ is determined by the resonance condition (54) and the group velocity of the resonant radiation is given by the $k$ derivative of the short wave dispersion relation (52). The resonant radiation is a solution of the nematic equations in the limit $v k^{2} \gg 1$, so that the appropriate group velocity for it is that from the dispersion relation (52), not the linearized KdV group velocity of the eKdV Equation (42) [36].

Figure 6 displays comparisons of the nematic DSW amplitude $a_{S}$ and resonant wave amplitude $a_{r}$ in the CDSW regime as given by (68) and (70) and numerical solutions. A typical CDSW is shown in Figure 2c, with details of the actual CDSW of this figure shown in Figure 7a. It can be seen that the lead waves of the DSW have an approximately uniform amplitude, with a rapid decrease of the amplitude towards the trailing edge of the CDSW, as also illustrated in Figure 7b. This solution, and that of Figure 7a, are typical structures for an unstable DSW [56]. The numerical DSW amplitude for the comparisons of this figure was calculated as an average over the approximately uniform waves at the leading edge, which is the same assumption on which the equal amplitude approximation used to calculate the solitary wave amplitude $a_{s}$ was based. Figure $6 \mathrm{a}$ shows comparisons for the amplitude $a_{r}$ of the resonant wavetrain leading the CDSW; see Figure 2c. It can be seen that there is excellent agreement between the theoretical amplitude and the numerical amplitude for the larger values of the level ahead $u_{+}$, with the increase of $a_{r}$ as the nonlocality parameter $v$ decreases being correctly given. This agreement is much improved through the inclusion of the all the higher order terms in the eKdV Equation (42) than that of previous work [36] based on the Kawahara equation, for which $c_{1}=c_{2}=c_{3}=0$, as given by the green dashed line in the figure. As $u_{+}$decreases and the TDSW regime is approached, the agreement between theory and numerical solutions decreases. This is shown particularly in the final comparison of Figure 6a for $u_{+}=0.5$, which is near the TDSW boundary; see Figure 3. The reason for this decreasing agreement is that, as the TDSW regime is approached, the number of waves in the CDSW decreases so that only one lead wave is left; see Figure 7c. The approximation that an average can be taken over an equal amplitude wavetrain then breaks down. 

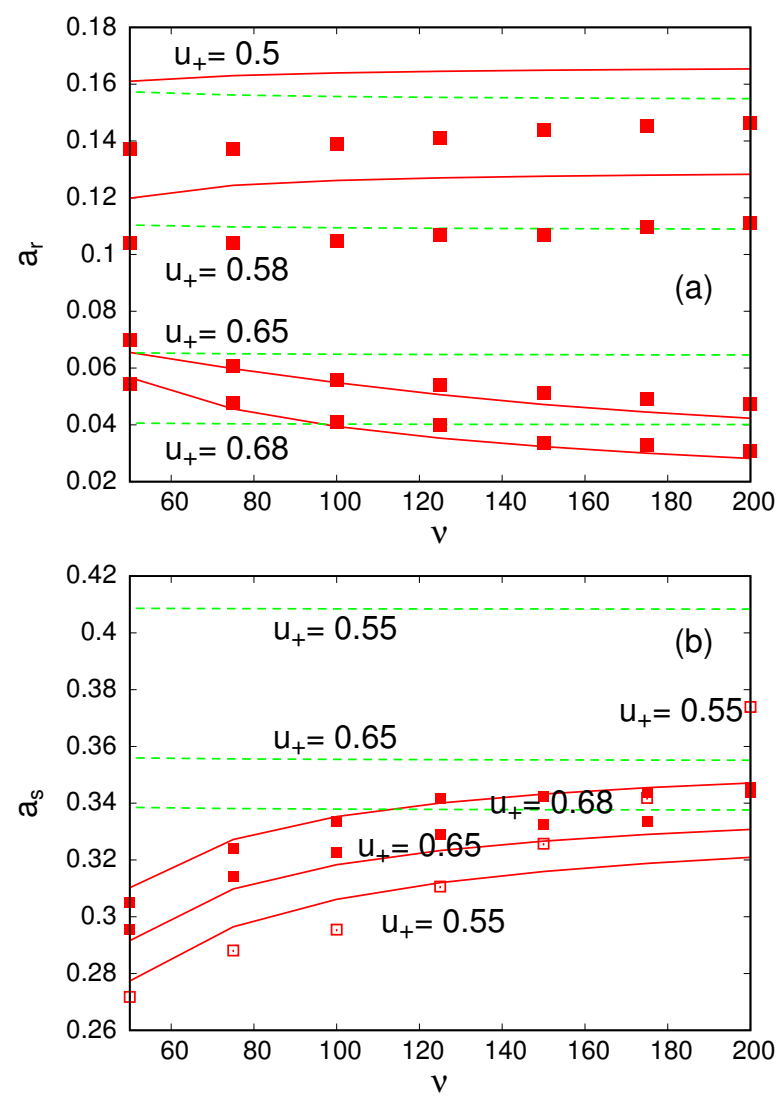

Figure 6. Comparison between theory and numerical solutions of the nematic Equations (11) and (12) with the initial condition (13) for the nematic CDSW. Numerical amplitude: red squares; amplitude (70): red (solid) line; amplitude given by the Kawahara equation (equation (42) with $c_{1}=c_{2}=c_{3}=0$ ): green (dashed) line. (a) Resonant wave amplitude $a_{r}$, (b) CDSW solitary wave amplitude $a_{S}$ (67). Note that, for clarity, the numerical DSW amplitude for $u_{+}=0.55$ is denoted by red open squares. Here, $u_{-}=1.0$ and $q=2$.

Figure $6 \mathrm{~b}$ displays similar comparisons for the amplitude $a_{s}$ of the nematic CDSW with numerical solutions. It can be seen that the comparison for the DSW amplitude is similar to that for the resonant wave amplitude. It should be noted that different values of $u_{+}$have been chosen for the DSW amplitude comparisons for the sake of clarity. The inclusion of all the higher order terms in the eKdV Equation (42) results in a significant improvement in the agreement with numerical solutions over that based on the Kawahara equation with $c_{1}=c_{2}=c_{3}=0$ when the level ahead $u_{+}$is away from the TDSW /CDSW boundary of Figure 3. As $u_{+}$approaches the TDSW/CDSW boundary, the DSW amplitude as given by the eKdV equation differs significantly from the numerical amplitude. The reason for this is that discussed in the previous paragraph for the resonant wave amplitude, the fact that the CDSW ceases to be a train of equal amplitude solitary waves, but reduces to a few waves. 

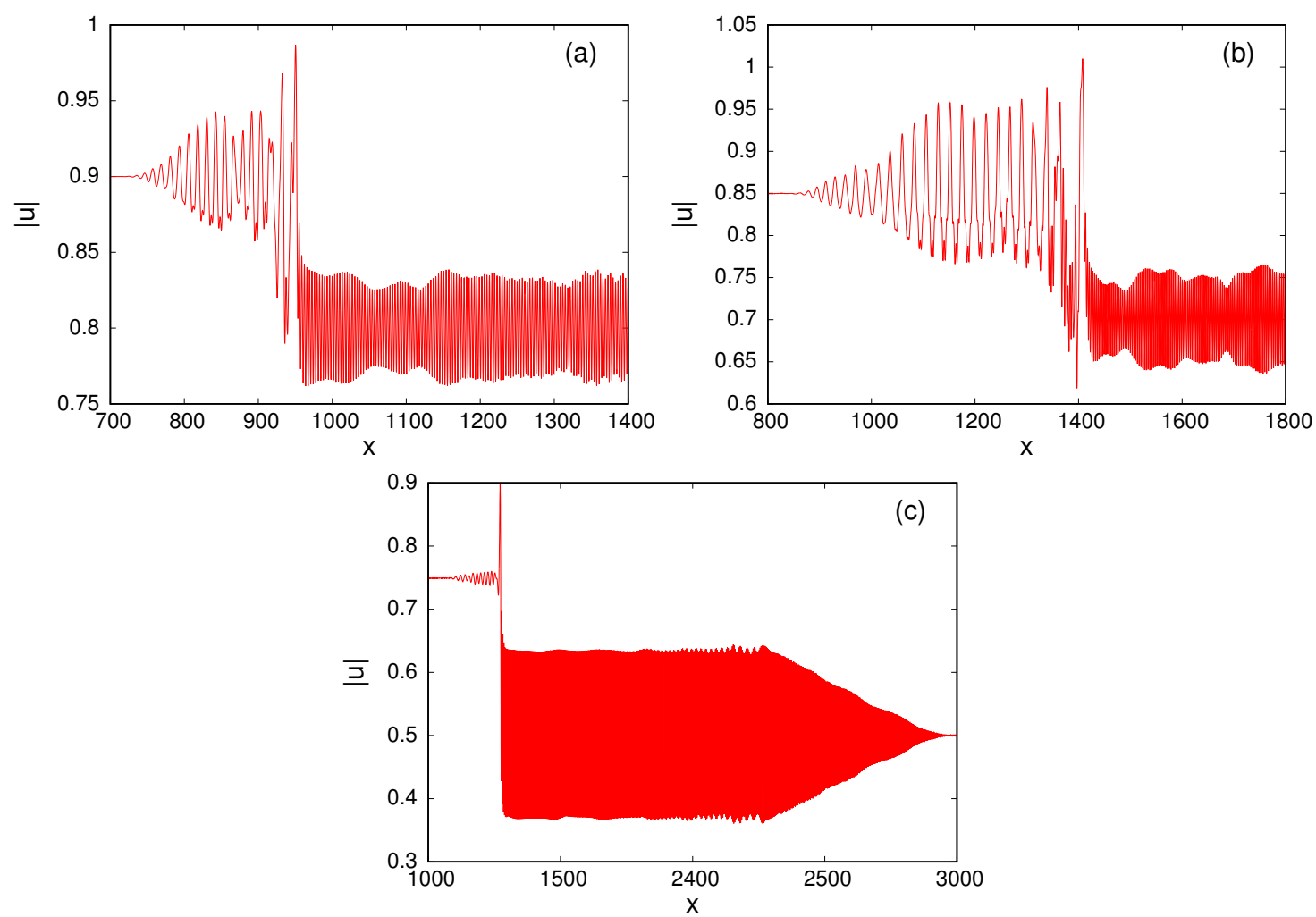

Figure 7. Numerical solution of nematic Equations (11) and (12) in the CDSW regime for the initial condition (13). (a) $u_{+}=0.8$ at $z=1000,(\mathbf{b}) u_{+}=0.7$ at $z=1500,(\mathbf{c}) u_{+}=0.5$ at $z=1500$. Here, $u_{-}=1.0$ and $q=2$.

The next thing that we discuss is the analytical borderline between the nematic CDSW and RDSW regimes. This borderline can be found by determining when the resonant amplitude (70) approaches zero or approaches a minimum as a function of the nonlocality parameter $v$. The determination of this borderline is similar to that of previous work [36] in which the resonant amplitude was found as a function of the initial state $u_{+}$, rather than $v$. This approach gave a borderline for $u_{+} \leq 0.73$ for large $v$, with the CDSW regime not existing above this value of $u_{+}$for large $v$. Above this limit, the resonance condition (53) ceases to work as a function of $v$ and an alternative method needs to be found to determine the borderline. To determine the borderline in this case, we exploit the fact that the (interior) structure of the nematic DSW is resonant, as evidenced in Figure 5, not only the leading, solitary wave edge. A DSW is an extended modulated periodic wavetrain, so that all its component waves can resonant, not just the leading edge. To verify this internal resonance, the phase velocity of the modulated cnoidal waves forming the DSW needs to be matched with the nematic linear phase velocity on the local mean level of the DSW. By way of illustration, we equate the DSW phase velocity [47]

$$
\begin{aligned}
c_{p}=\sqrt{\frac{2}{q} u_{+}+} & \frac{\left(u_{i}-u_{+}\right)}{\sqrt{2 q}}\left\{2+2 m-\frac{1}{7}\left(u_{i}-u_{+}\right)\left(8 C_{4}-C_{3}\right)\left(2 m-m^{2}-1\right)\right. \\
& \left.+\frac{1}{3}\left(u_{i}-u_{+}\right) C_{4}(2+2 m)^{2}-\frac{1}{6}\left(u_{i}-u_{+}\right)\left(C_{3}-C_{1}+4 C_{4}\right)(2+2 m)\right\},
\end{aligned}
$$

where $C_{1}, C_{3}$, and $C_{4}$ are given in (49), with the nematic phase velocity from the full linear dispersion relation (51) on the DSW background (47) and solved for internal resonant wavenumbers, which are always positive and real, as the modulus $m$ varies from near zero to near one. As the DSW parameter expressions (47) and (72) are only valid for a well-ranked DSW (stable DSW), such as the PDSW and the RDSW, and the CDSW is an ill-ranked DSW (unstable DSW), then a borderline exists when these wave parameter 
expressions result in imaginary internal resonant wavenumbers, which are unphysical, at a borderline value of $v$. The mean flow $\bar{v}$ in the dispersion relation (51) was determined from the extended $\mathrm{KdV}$ reduction of the nematic equations of Section 4. Substituting the $O\left(\varepsilon^{2}\right)$ mean flow $v_{1}$ given by (31) into the mean flow perturbation expansion (27) for $v$ and then averaging gives

$$
\bar{v}=\frac{2 \sqrt{2}}{\sqrt{q}}\left(|\bar{u}|-u_{+}\right) .
$$

This then completes the determination of the CDSW / RDSW borderline. A comparison between the theoretical and numerical borderlines between the RDSW and CDSW regimes is shown in Figure 3. It can be seen that the theoretical borderline is in excellent agreement with the numerical borderline for large values of the nonlocality parameter $v$ down to around $v=50$, with poorer agreement towards the local limit with $v$ small for which the DSW is changing form from KdV type to NLS type.

\section{TDSW Regime}

Figure $2 \mathrm{~d}$ displays a typical DSW in the TDSW regime. There is (almost) a constant amplitude resonant wavetrain which, at its trailing edge, is connected to the intermediate level $u_{i}$. At its leading edge there is a modulated wavetrain which takes $u$ down to the level $u_{+}$ahead. This wavetrain leading the resonant wave is a partial DSW [27,36,49]. A partial DSW differs from a standard DSW in that it connects a uniform state to a periodic wavetrain, unlike a standard DSW which links two different levels. While there is a negative polarity solitary wave connecting the resonant wavetrain to the intermediate level [26], this connection can be approximated by a Whitham shock [36,48], a shock, a jump, in the modulation parameters of the wavetrain, wavelength, frequency, amplitude, and mean level, of the Whitham modulation equations for the modulated periodic wavetrain [12]. A Whitham shock is determined from the Whitham modulation equations. As noted above, the nematic DSW is in the nonlocal regime, so the appropriate Whitham modulation equations are those for $v$ large.

The Whitham modulation equations in the highly nonlocal limit $v \gg 1$ (low optical power) have previously been determined [36], so these modulation equations will just be quoted here. These modulation equations determine the mean level $\bar{\rho}$ of $\rho$, the amplitude $a$ and the wavenumber $k$ of the Stokes' wave solution of the nematic Equations (15)-(17). As there is no known general periodic wave solution of the nematic equations, the highly nonlocal Whitham modulation equations are derived based on the weakly nonlinear Stokes wavetrain for the nematic equations [36]. It can be seen from Figure $2 \mathrm{~d}$ that the resonant wavetrain has small amplitude, so the weakly nonlinear limit is appropriate. In the highly nonlocal limit $v \gg 1$, the Stokes' wave solution of the nematic Equations (15)-(17) is

$$
\begin{aligned}
\rho & =\bar{\rho}+a \cos \varphi+\ldots \\
v & =\bar{v}+a v_{1} \cos \varphi+\ldots \\
\phi & =\frac{\bar{\rho}}{q}+a \phi_{1} \cos \varphi+\ldots \\
\omega & =\omega_{0}+a \omega_{1}+a^{2} \omega_{2}+\ldots
\end{aligned}
$$

where the uniform phase is $\varphi=k x-\omega z$, and the over bar, $\bar{\rho}$ and $\bar{v}$, denotes the mean value of a wave parameter [36]. The amplitude $a$ of the (Stokes) wave is assumed to be 
small. The work of Reference [36] gives that, at $O(a)$, the nematic Stokes coefficients and the $O\left(a^{2}\right)$ correction to the dispersion relation are

$$
\begin{aligned}
\omega_{0} & =k \bar{v}+\frac{k^{2}}{2}+\frac{4 \bar{\rho}}{v k^{2}}-\frac{8 \bar{\rho} q}{v^{2} k^{4}}-\frac{16 \bar{\rho}^{2}}{v^{2} k^{6}}+\ldots, \\
v_{1} & =\frac{k}{2 \bar{\rho}}+\frac{4}{v k^{3}}-\frac{8 q}{v^{2} k^{5}}-\frac{16 \bar{\rho}}{v^{2} k^{7}}+\ldots, \\
\phi_{1} & =\frac{2}{v k^{2}}-\frac{4 q}{v^{2} k^{4}}+\ldots, \\
\omega_{2} & =-\frac{k^{2}}{8 \bar{\rho}^{2}}-\frac{3}{\bar{\rho} v k^{2}}+\frac{6 q}{\bar{\rho} v^{2} k^{4}}-\frac{20}{v^{2} k^{6}}+\ldots
\end{aligned}
$$

The coefficient $\omega_{1}$ is set to zero, $\omega_{1}=0$, to eliminate secular terms. The weakly nonlinear Whitham modulation equations can then be derived by averaging conservation laws deduced from Nöther's Theorem [59]. The Lagrangian for the hydrodynamic form of the nematic Equations (15)-(17) is

$$
L=-2 \rho \psi_{z}-\frac{1}{4} \frac{\rho_{x}^{2}}{\rho}-\rho \psi_{x}^{2}-4 \rho \phi+v \phi_{x}^{2}+2 q \phi^{2} .
$$

Applying Nöther's Theorem we have that translation invariance with respect to the phase $\psi$ gives the mass conservation law

$$
\frac{\partial}{\partial z} \frac{\partial L}{\partial \psi_{z}}+\frac{\partial}{\partial x} \frac{\partial L}{\partial \psi_{x}}=0
$$

translation invariance with respect to space $x$ yields the momentum conservation law

$$
\frac{\partial}{\partial z}\left(\frac{\partial L}{\partial \rho_{z}} \frac{\partial \rho}{\partial x}+\frac{\partial L}{\partial \psi_{z}} \frac{\partial \psi}{\partial x}+\frac{\partial L}{\partial \phi_{z}} \frac{\partial \phi}{\partial x}\right)+\frac{\partial}{\partial x}\left(\frac{\partial L}{\partial \rho_{x}} \frac{\partial \rho}{\partial x}+\frac{\partial L}{\partial \psi_{x}} \frac{\partial \psi}{\partial x}+\frac{\partial L}{\partial \phi_{x}} \frac{\partial \phi}{\partial x}-L\right)=0,
$$

and translation invariance with respect to time-like $z$ gives the energy conservation law

$$
\frac{\partial}{\partial z}\left(\frac{\partial L}{\partial \rho_{z}} \frac{\partial \rho}{\partial z}+\frac{\partial L}{\partial \psi_{z}} \frac{\partial \psi}{\partial z}+\frac{\partial L}{\partial \phi_{z}} \frac{\partial \phi}{\partial z}-L\right)+\frac{\partial}{\partial x}\left(\frac{\partial L}{\partial \rho_{x}} \frac{\partial \rho}{\partial z}+\frac{\partial L}{\partial \psi_{x}} \frac{\partial \psi}{\partial z}+\frac{\partial L}{\partial \phi_{x}} \frac{\partial \phi}{\partial z}\right)=0 .
$$

Substituting the Stokes expansions (74)-(76) into these conservation laws and averaging by integrating in $\varphi$ from 0 to $2 \pi$ [12] yields the modulation equations, truncated to $O(1 / v)$,

$$
\begin{aligned}
& \frac{\partial k}{\partial z}+\frac{\partial}{\partial x}\left(k \bar{v}+\frac{k^{2}}{2}+\frac{2 \bar{\rho}}{q}+\frac{4 \bar{\rho}}{v k^{2}}-\frac{k^{2} a^{2}}{8 \bar{\rho}^{2}}-\frac{3 a^{2}}{v \bar{\rho} k^{2}}\right)=0 \\
& \frac{\partial \bar{\rho}}{\partial z}+\frac{\partial}{\partial x}\left(\bar{\rho} \bar{v}+\frac{k a^{2}}{4 \bar{\rho}}+\frac{2 a^{2}}{v k^{3}}\right)=0 \\
& \frac{\partial}{\partial z}\left(\bar{\rho} \bar{v}+\frac{k a^{2}}{4 \bar{\rho}}+\frac{2 a^{2}}{v k^{3}}\right)+\frac{\partial}{\partial x}\left(\frac{\bar{\rho}^{2}}{q}+\bar{\rho} \bar{v}^{2}+\frac{k^{2} a^{2}}{4 \bar{\rho}}+\frac{\bar{v} k a^{2}}{2 \bar{\rho}}+\frac{4 \bar{v} a^{2}}{v k^{3}}+\frac{a^{2}}{v k^{2}}\right)=0 \\
& \frac{\partial}{\partial z}\left(\bar{\rho} \bar{v}^{2}+\frac{2 \bar{\rho}^{2}}{q}+\frac{k^{2} a^{2}}{4 \bar{\rho}}+\frac{k \bar{v} a^{2}}{2 \bar{\rho}^{2}}+\frac{4 a^{2}}{v k^{2}}+\frac{4 \bar{v} a^{2}}{v k^{3}}\right)+\frac{\partial}{\partial x}\left(\bar{\rho} \bar{v}^{3}+\frac{4 \bar{v} \bar{\rho}^{2}}{q}+\frac{k a^{2}}{q}+\frac{k^{3} a^{2}}{4 \bar{\rho}}\right. \\
& \left.+\frac{3 \bar{v} k^{2} a^{2}}{4 \bar{\rho}}+\frac{3 \bar{v}^{2} k a^{2}}{4 \bar{\rho}}+\frac{6 \bar{v} a^{2}}{v k^{2}}+\frac{6 \bar{v}^{2} a^{2}}{v k^{3}}+\frac{2 a^{2}}{v k}\right)=0
\end{aligned}
$$

for the (slowly varying) amplitude $a$, wavenumber $k$, and means $\bar{\rho}$ and $\bar{v}$ of the modulated Stokes wave [12].

The modulation Equation (87) is that, for optical power conservation, Equation (88) is momentum conservation, and (89) is energy conservation. The modulation Equation (86) 
is the equation for conservation of waves, $k_{z}+\omega_{x}=0$, on noting that the $x$ derivative term is just the dispersion relation (77). In this regard, it should be noted that the dispersion relation for the Stokes' wave from which the modulation equations are calculated has the carrier waves' phase shift term $2 \bar{\rho} / q$ added $[35,36]$, as explained above,

$$
\omega=k \bar{v}+\frac{1}{2} k^{2}+\frac{2 \bar{\rho}}{q}+\frac{4 \bar{\rho}}{v k^{2}}-\frac{k^{2} a^{2}}{8 \bar{\rho}^{2}}-\frac{3 a^{2}}{v k^{2} \bar{\rho}} .
$$

Figure 2d shows that, in the TDSW regime, the KdV-type nematic bore structure of Figure 2a-c has disappeared, leaving a dominant resonant wavetrain which is linked to the intermediate level $u_{i}$ by a negative polarity solitary wave [26]. As discussed above, this link between the resonant wavetrain and the intermediate level can be approximated by a Whitham shock, a jump in the modulation equation variables [36,48], so that the Whitham shock links the resonant wavetrain with the level $u_{i}$ behind, in a similar manner to a gas dynamic shock wave links two compressible flow states [12]. Let us denote the amplitude, wavenumber, mean level and mean phase gradient of the resonant wavetrain by $a_{r}, k_{r}, \bar{\rho}_{r}$, and $\bar{v}_{r}$, respectively. Matching the Whitham shock velocity $U_{s}$ to the Stokes' wave velocity (90), as these are co-propagating, gives

$$
U_{s}=\bar{v}_{r}+\frac{1}{2} k_{r}+\frac{2 \bar{\rho}_{r}}{q k_{r}}+\frac{4 \bar{\rho}_{r}}{v k_{r}^{3}}-\frac{k_{r} a_{r}^{2}}{8 \bar{\rho}_{r}^{2}}-\frac{3 a_{r}^{2}}{v k_{r}^{3} \bar{\rho}_{r}} .
$$

Ahead of the Whitham shock, there is the resonant wavetrain, and behind it is a flat shelf, the intermediate level, which is a wavetrain of zero amplitude. The mass, momentum, and energy conservation Equations (87)-(89) then give the jump conditions

$$
\begin{aligned}
& U_{s}\left(\bar{\rho}_{r}-\rho_{i}\right)=\bar{\rho}_{r} \bar{v}_{r}+\frac{k_{r} a_{r}^{2}}{4 \bar{\rho}_{r}}+\frac{2 a_{r}^{2}}{v k_{r}^{3}}-\rho_{i} v_{i}, \\
& U_{s}\left(\bar{\rho}_{r} \bar{v}_{r}+\frac{k_{r} a_{r}^{2}}{4 \bar{\rho}_{r}}+\frac{2 a_{r}^{2}}{v k_{r}^{3}}-\rho_{i} v_{i}\right)=\frac{\bar{\rho}_{r}^{2}}{q}+\bar{\rho}_{r} \bar{v}_{r}^{2}+\frac{k_{r}^{2} a_{r}^{2}}{4 \bar{\rho}_{r}}+\frac{\bar{v}_{r} k_{r} a_{r}^{2}}{2 \bar{\rho}_{r}}+\frac{4 \bar{v}_{r} a_{r}^{2}}{v k_{r}^{3}} \\
& +\frac{a_{r}^{2}}{v k_{r}^{2}}-\frac{\rho_{i}^{2}}{q}-\rho_{i} v_{i}^{2}, \\
& U_{S}\left(\bar{\rho}_{r} \bar{v}_{r}^{2}+\frac{2 \bar{\rho}_{r}^{2}}{q}+\frac{k_{r}^{2} a_{r}^{2}}{4 \bar{\rho}_{r}}+\frac{k_{r} \bar{v}_{r} a_{r}^{2}}{2 \bar{\rho}_{r}}+\frac{4 a_{r}^{2}}{v k_{r}^{2}}+\frac{4 \bar{v}_{r} a_{r}^{2}}{v k_{r}^{3}}-\rho_{i} v_{i}^{2}-\frac{2 \rho_{i}^{2}}{q}\right)=\bar{\rho}_{r} \bar{v}_{r}^{3}+\frac{4 \bar{v}_{r} \bar{\rho}_{r}^{2}}{q} \\
& +\frac{k_{r} a_{r}^{2}}{q}+\frac{k_{r}^{3} a_{r}^{2}}{4 \bar{\rho}_{r}}+\frac{3 \bar{r}_{r} k_{r}^{2} a_{r}^{2}}{4 \bar{\rho}_{r}}+\frac{3 \bar{s}_{r}^{2} k_{r} a_{r}^{2}}{4 \bar{\rho}_{r}}+\frac{6 \bar{v}_{r} a_{r}^{2}}{v k_{r}^{2}}+\frac{6 \bar{v}_{r}^{2} a_{r}^{2}}{v k_{r}^{3}}+\frac{2 a_{r}^{2}}{v k_{r}}-\rho_{i} v_{i}^{3}-\frac{4 v_{i} \rho_{i}^{2}}{q}
\end{aligned}
$$

across the Whitham shock. Together with the resonance condition (91), these form four equations for the five unknowns $U_{s}, a_{r}, k_{r}, \bar{\rho}_{r}$, and $\bar{v}_{r}$, noting that $\rho_{i}$ and $v_{i}$ are given by (24) and (25), respectively. The final equation is obtained by assuming that the Riemann invariant $R_{-}$(22) is conserved through the resonant wavetrain and its lead partial DSW, which is valid for a full DSW [19,50]. This then determines the mean of the resonant phase gradient $\bar{v}_{r}$ [36]. This Riemann invariant condition gives

$$
\bar{v}_{r}=2 \sqrt{\frac{2}{q}}\left(\sqrt{\bar{\rho}_{r}}-\sqrt{\rho_{+}}\right)=2 \sqrt{\frac{2}{q}}\left(\sqrt{\bar{\rho}_{r}}-u_{+}\right) .
$$

The above nematic Whitham modulation equation jump conditions (92)-(93) with (94) can be solved numerically for $U_{s}, a_{r}, k_{r}$, and $\bar{\rho}_{r}$ using Newton's method. The full details for this numerical solution of the Whitham shock jump conditions can be found in Reference [36]. Figure 8 shows comparisons for the Whitham shock velocity from the nonlocal to local limits, the optical power increasing, as given by the jump conditions and full numerical solutions of the nematic Equations (11) and (12). The values of the level ahead $u_{+}$were chosen to lie in the TDSW regime; see Figure 3. It can be seen that there is excellent agreement between the theoretical and numerical solutions from high nonlocality, $v$ large, down to $v=O(10)$. As for the lead solitary wave amplitude comparison of Figure 4, 
there is little change in the Whitham shock velocity as the nonlocality parameter $v$ varies by an order of magnitude, with only a small increase in the velocity. There is a small, increasing deviation between the theoretical and numerical values towards $v=20$. This is due to the onset on the VDSW regime for which $u$ vanishes at a point, a vacuum point $[35,36]$. Once the vacuum point is reached, $|u|$ cannot decrease further, so that the Whitham shock jump conditions need to be modified [36]. This will not be pursued further here.

For a fixed level ahead $u_{+}$, as the nonlocality parameter $v$ decreases (optical power increases), the nematic DSW evolves from CDSW to TDSW type; see Figure 3. The borderline between the CDSW and TDSW regimes can be determined from the Whitham shock jump conditions (92)-(93) and the resonance condition (91) based on the following condition. For a fixed nonlocality parameter $v$, as the level ahead $u_{+}$increases, it is found that the Whitham shock velocity becomes greater than the linear group velocity

$$
c_{g}=\bar{v}_{r}+k_{r}-\frac{8 \bar{\rho}_{r}}{v k_{r}^{3}}
$$

of the resonant wavetrain. This is unphysical as this would mean that the resonant wavetrain could not form. Figure 3 shows this theoretical bound between the CDSW and TDSW regimes as a red line. It can be seen that the agreement with numerical solutions is excellent over the entire range of $v$, even for jump heights $u_{-}-u_{+}$, which are not small.

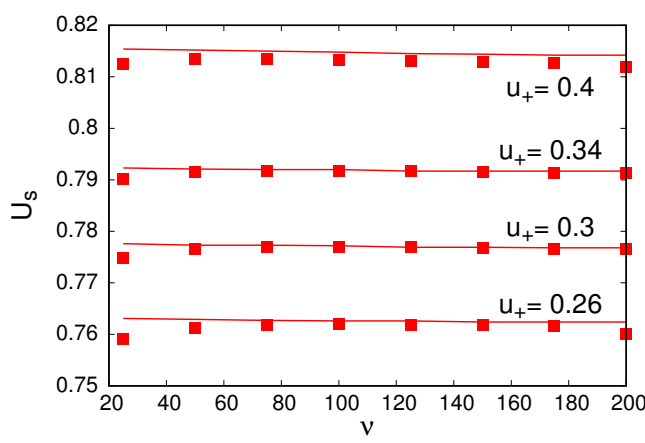

Figure 8. Comparison between the Whitham shock velocity $U_{s}$ given by the modulation equation jump conditions (92)-(93) with (94) and numerical solutions of the nematic Equations (11) and (12) in the TDSW regime. Solution of jump conditions: red (full) line; numerical solution: red boxes. Here, $u_{-}=1.0$ and $q=2$.

As $v$ decreases, it would be expected that the high nonlocality modulation Equations (86)-(89) cease to be applicable. The Whitham modulation equations for the nematic equations in the local limit, $v$ small, were calculated based on the equivalent of the Stokes' wave expansions (74)-(77) and (78)-(81), expanding in $v$ rather than $1 / v$. However, these were found not to give solutions in good agreement with numerical solutions. This is expected as Figures 3 and 4 show that the nematic DSW is highly nonlocal down to very small values of $v$, which are unphysical, as discussed at the end of Section 3.

\section{RNLS and NLS DSW Regimes}

The KdV approximation (44) gives that the DSW changes from KdV to NLS type at $v=$ $q^{2} /\left(4 u_{+}^{2}\right)$ due to the change in sign of the coefficient of the third derivative, as discussed above, and as shown in Figure 3. It should be noted that numerical solutions do not show a distinct change in DSW type, but a transition from a KdV-type DSW to an NLS-type DSW, as seen in Figure 2d,e and as shown by the two regimes RNLS and NLS type in Figure 3. The TDSW regime is characterized by a negative polarity solitary wave connecting the resonant wavetrain to the intermediate level, as in Figure 2d. As the nonlocality $v$ decreases, the beam power in increases, and the height of this solitary wave decreases, resulting in the RNLS regime which consists of a Whitham shock connecting a resonant wavetrain to the intermediate level $u_{i}$. Ahead of the resonant wavetrain is a partial NLS-type DSW 
which connects to the level ahead $u_{+}$; see Figure 2e. As $v$ decreases further, the resonant wavetrain shrinks, and the partial DSW becomes a full NLS DSW with solitary waves at its trailing edge and linear waves at its leading edge, resulting in an NLS DSW for sufficiently small v; see Figure $3 \mathrm{~b}$. In this limit, the NLS DSW alone links the intermediate level to the level ahead. As $v$ decreases in the RNLS regime, the waves at the trailing edge of the partial DSW evolve from weakly nonlinear Stokes waves to fully nonlinear periodic waves, and then solitary waves in the NLS DSW regime.

The intermediate RNLS state, illustrated in Figure 2e, consists of a resonant wavetrain with the height of the negative polarity solitary wave at the Whitham shock negligible. To compare the KdV-NLS DSW boundary $v=q^{2} /\left(4 u_{+}^{2}\right)$ with numerical solutions, the choice of the height of this solitary wave being $5 \times 10^{-3}$ above $u_{i}$ was chosen for the onset of the RNLS regime in numerical solutions. It can be seen that there is good agreement for this regime boundary for $u_{+}$close to $u_{-}$, but there is increasing disagreement as $u_{+}$decreases. This is expected as the reductive nematic eKdV Equation (42) was derived under the assumption that $\left|u_{-}-u_{+}\right|$is small.

\section{Conclusions}

The structure of the nematic DSW (dispersive shock wave) solution of the defocusing nematic equations governing the propagation of an optical beam through a cell filled with nematic liquid crystals has been investigated using a combination of numerical solutions of the equations governing the beam, consisting of an NLS-type equation for the optical beam and an elliptic medium response equation, and solutions of the governing nematic equations using Whitham modulation theory and/or asymptotic solutions. In contrast to previous work [34-36], the evolution of the DSW structure was studied as the power of the optical beam varied, from the experimental low power for which the nematic response is nonlocal to high power for which the response is local. As the beam power varies, it was found that the nematic DSW transitions between six regimes, four of which were studied in previous work [34-36]. The two NLS-type DSWs do not exist in the low power regime studied in this previous work. However, the experimental verification of these high power DSW types is questionable as the powers required for their existence are unrealistic due to the possible excessive medium heating the high beam powers would cause. Excellent agreement was found between numerical solutions and analytical solutions for the four physically relevant DSW regimes, the PDSW, RDSW, CDSW, and TDSW regimes displayed in Figure 2a-d. In particular, the analytical theory gives good agreement for the boundaries between the existence regions for five of the DSW types as the nonlocality parameter $v$ varies, the exception being the boundary between the PDSW and RDSW regimes.

It has been found that the details of the nematic DSW, for instance, its lead wave amplitude and velocity and the amplitude of the associated resonant radiation, are well approximated by the nematic equations in the high nonlocality limit, the nonlocality parameter $v$ large, as the DSW transitions to the local limit. This holds for the PDSW, RDSW, CDSW and TDSW regimes with the nonlocality parameter $v$ ranging from $O(100)$ to $O(1)$. As the analysis of the nematic DSW is much easier in the high nonlocality limit based on asymptotic analyses with $v \gg 1$, this is an important result for future analysis of the nematic DSW in its various regimes and over its nonlocality range.

There are still a number of issues which could be addressed by future work. An outstanding issue is the correct determination of resonance between the nematic DSW and diffractive radiation. In contrast to the high nonlocality limit with $v$ large [34-36], as $v$ decreases from the high to the low nonlocality limit, the beam power increases, and the resonance condition used in previous work in the RDSW and CDSW regimes that the velocity of the lead solitary wave of the DSW matches the linear phase velocity of the resonant waves does not agree with numerical solutions. The theoretical transition from the PDSW regime for high nonlocality to the RDSW regime due to the onset of resonance as $v$ decreases occurs for $v=O(1)$, while numerical solutions show the transition for $v=O(100)$ to $O(10)$. As noted in previous work [36], and in contrast with other work 
on resonant DSWs, not only is the lead wave of the DSW in resonance with diffractive radiation, but the whole modulated periodic wave which forms the DSW is in resonance. However, even this observation does not yield the correct resonance condition for the RDSW regime as the nonlocality parameter $v$ decreases below the highly nonlocal limit. The correct resonance condition between the DSW and diffractive radiation requires further study. This should be an important general issue for all resonant DSWs beyond the specific application to nematic liquid crystals.

Author Contributions: Conceptualization, S.B., D.J.F., T.P.H., C.H., T.R.M. and N.F.S.; methodology, S.B., D.J.F., T.P.H., C.H., T.R.M. and N.F.S.; software, S.B., T.R.M. and N.F.S.; validation, S.B., D.J.F., T.P.H., C.H., T.R.M. and N.F.S.; methodology, S.B., D.J.F., T.P.H., C.H., T.R.M. and N.F.S.; formal analysis, S.B., D.J.F., T.P.H., C.H., T.R.M. and N.F.S.; methodology, S.B., D.J.F., T.P.H., C.H., T.R.M. and N.F.S.; investigation, S.B., D.J.F., T.P.H., C.H., T.R.M. and N.F.S.; methodology, S.B., D.J.F., T.P.H., C.H., T.R.M. and N.F.S.; writing-S.B., D.J.F., T.P.H., C.H., T.R.M. and N.F.S.; methodology, S.B., D.J.F., T.P.H., C.H., T.R.M. and N.F.S.; writing-review and editing, S.B., D.J.F., T.P.H., C.H., T.R.M. and N.F.S.; methodology, S.B., D.J.F., T.P.H., C.H., T.R.M. and N.F.S.; project administration, N.F.S. All authors have read and agreed to the published version of the manuscript.

Funding: This research received no external funding.

Data Availability Statement: There is no experimental data resulting from this research. The numerical data is available on request from the authors.

Acknowledgments: Saleh Baqer thanks the research sector of Kuwait University for a Research Initiation Grant (RIG) given during the preparation of the paper.

Conflicts of Interest: The authors declare no conflict of interest.

\section{References}

1. Khoo, I.C. Liquid Crystals: Physical Properties and Nonlinear Optical Phenomena; Wiley: New York, NY, USA, 1995.

2. Peccianti, M.; Assanto, G. Nematicons. Phys. Rep. 2012, 516, 147-208. [CrossRef]

3. Assanto, G. Nematicons: Reorientational solitons from optics to photonics. Liq. Cryst. Rev. 2018, 6, 170-194. [CrossRef]

4. Conti, C.; Peccianti, M.; Assanto, G. Route to nonlocality and observation of accessible solitons. Phys. Rev. Lett. 2003, 91, 073901. [CrossRef] [PubMed]

5. Assanto, G.; Smyth, N.F. Self-confined light waves in nematic liquid crystals. Phys. D 2020, 402, 132182. [CrossRef]

6. Peccianti, M.; Assanto, G.; Luca, A.D.; Umeton, C.; Khoo, I.C. Electrically assisted self-confinement and waveguiding in planar nematic liquid crystal cells. Appl. Phys. Lett. 2000, 77, 7-9. [CrossRef]

7. Peccianti, M.; Assanto, G. Signal readdressing by steering of spatial solitons in bulk nematic liquid crystals. Opt. Lett. 2001, 26, 1690-1692. [CrossRef]

8. Assanto, G.; Peccianti, M. Spatial solitons in nematic liquid crystals. IEEE J. Quantum Electron. 2003, 39, 13-21. [CrossRef]

9. Piccardi, A.; Alberucci, A.; Bortolozzo, U.; Residori, S.; Assanto, G. Readdressable interconnects with spatial soliton waveguides in liquid crystal light valves. IEEE Photon. Technol. Lett. 2010, 22, 694-696. [CrossRef]

10. Karimi, N.; Virkki, M.; Alberucci, A.; Buchnev, O.; Kauranen, M.; Priimagi, A.; Assanto, G. Molding optical waveguides with nematicons. Adv. Opt. Mater. Commun. 2017, 5, 1700199. [CrossRef]

11. Assanto, G.; Minzoni, A.A.; Smyth, N.F. Light self-localization in nematic liquid crystals: Modelling solitons in nonlocal reorientational media. J. Nonlin. Opt. Phys. Mater. 2009, 18, 657-691. [CrossRef]

12. Whitham, G.B. Linear and Nonlinear Waves; J. Wiley and Sons: New York, NY, USA, 1974.

13. Russell, J.S. Repoerrt of the Fourteen Meeting of the British Association for the Advancement of Science, Report on Waves; The British Association for the Advancement of Science: London, UK, September 1844; pp. 311-390.

14. Hooper, C.G.; Ruiz, P.D.; Huntley, J.M.; Khusnutdinova, K.R. Undular bores generated by fracture. Phys. Rev. E 2021, submitted.

15. Davydov, A.S. Solitons in Molecular Systems, 2nd ed.; Kluwer Academic Publishers: Dordrecht, The Netherlands, 1991.

16. Tlidi, M.; Staliunas, K.; Panajotov, K.; Vladimirov, A.G.; Clerc, M.G. Localized structures in dissipative media: From optics to plant ecology. Phil. Trans. R. Soc. A 2014, 372, 20140101. [CrossRef] [PubMed]

17. Kivshar, Y.S.; Agrawal, G.P. Optical Solitons. From Fibers to Photonic Crystals; Academic Press: San Diego, CA, USA, 2003.

18. Ablowitz, M.J. Nonlinear Dispersive Waves. Asymptotic Analysis and Solitons; Cambridge University Press: Cambridge, UK, 2011.

19. El, G.A.; Hoefer, M.A. Dispersive shock waves and modulation theory. Phys. D 2016, 333, 11-65. [CrossRef]

20. Whitham, G.B. A general approach to linear and non-linear dispersive waves using a Lagrangian. J. Fluid Mech. 1965, 22, 273-283. [CrossRef]

21. Whitham, G.B. Non-linear dispersive waves. Proc. Roy. Soc. Lond. A 1965, 283, 238-261.

22. Whitham, G.B. Variational methods and applications to water waves. Proc. Roy. Soc. Lond. A 1967, $299,6-25$. 
23. Gurevich, A.V.; Pitaevskii, L.P. Nonstationary structure of a collisionless shock wave. Sov. Phys. JETP 1974, 33, $291-297$.

24. Fornberg, B.; Whitham, G.B. Numerical and theoretical study of certain non-linear wave phenomena. Phil. Trans. Roy. Soc. Lond. Ser. A Math. Phys. Sci. 1978, 289, 373-404.

25. Flaschka, H.; Forest, M.G.; McLaughlin, D.W. Multiphase averaging and the inverse spectral solution of the Korteweg-de Vries equation. Comm. Pure Appl. Math. 1980, 33, 739-784. [CrossRef]

26. Sprenger, P.; Hoefer, M.A. Shock waves in dispersive hydrodynamics with nonconvex dispersion. SIAM J. Appl. Math. 2017, 77, 26-50. [CrossRef]

27. Hoefer, M.A.; Smyth, N.F.; Sprenger, P. Modulation theory solution for nonlinearly resonant, fifth-order Korteweg-de Vries, nonclassical, travelling dispersive shock waves. Stud. Appl. Math. 2019, 142, 219-240. [CrossRef]

28. Kawahara, T. Oscillatory solitary waves in dispersive media. J. Phys. Soc. Jpn. 1972, 33, 260-264. [CrossRef]

29. Conforti, M.; Trillo, S. Dispersive wave emission from wave breaking. Opt. Lett. 2013, 38, 3815-3818. [CrossRef] [PubMed]

30. Conforti, M.; Trillo, S. Radiative effects driven by shock waves in cavity-less four-wave mixing combs. Opt. Lett. 2014, 39, 5760-5763. [CrossRef]

31. Conforti, M.; Trillo, S.; Mussot, A.; Kudlinski, A. Parametric excitation of multiple resonant radiations from localized wavepackets. Sci. Rep. 2015, 5, 1-5. [CrossRef] [PubMed]

32. Malaguti, S.; Conforti, M.; Trillo, S. Dispersive radiation induced by shock waves in passive resonators. Opt. Lett. 2014, 39, 5626-5629. [CrossRef]

33. Piccardi, A.; Alberucci, A.; Tabiryan, N.; Assanto, G. Dark nematicons. Opt. Lett. 2011, 36, 1356-1358. [CrossRef]

34. Smyth, N.F. Dispersive shock waves in nematic liquid crystals. Phys. D 2016, 333, 301-309. [CrossRef]

35. El, G.A.; Smyth, N.F. Radiating dispersive shock waves in non-local optical media. Proc. Roy. Soc. Lond. A 2016, $472,20150633$. [CrossRef]

36. Baqer, S.; Smyth, N.F. Modulation theory and resonant regimes for dispersive shock waves in nematic liquid crystals. Phys. D 2020, 403, 132334. [CrossRef]

37. El, G.A.; Geogjaev, V.V.; Gurevich, A.V.; Krylov, A.L. Decay of an initial discontinuity in the defocusing NLS hydrodynamics. Phys. D 1995, 87, 186-192. [CrossRef]

38. Marchant, T.R.; Smyth, N.F. The initial-boundary problem for the Korteweg-de Vries equation on the negative quarter-plane. Proc. Roy. Soc. Lond. A 2002, 458, 857-871. [CrossRef]

39. García-Reimbert, C.; Minzoni, A.A.; Smyth, N.F.; Worthy, A.L. Large-amplitude nematicon propagation in a liquid crystal with local response. J. Opt. Soc. Am. B 2006, 23, 2551-2558. [CrossRef]

40. Assanto, G.; Minzoni, A.A.; Peccianti, M.; Smyth, N.F. Optical solitary waves escaping a wide trapping potential in nematic liquid crystals: Modulation theory. Phys. Rev. A 2009, 79, 033837. [CrossRef]

41. Izdebskaya, Y.; Krolikowski, W.; Smyth, N.F.; Assanto, G. Vortex stabilization by means of spatial solitons in nonlocal media. J. Opt. 2016, 18, 054006. [CrossRef]

42. Ghofraniha, N.; Conti, C.; Ruocco, G.; Trillo, S. Shocks in nonlocal media. Phys. Rev. Lett. 2007, 99, 043903. [CrossRef] [PubMed]

43. Kamchatnov, A.M. Whitham theory for perturbed Korteweg-de Vries equation. Phys. D 2016, 333, 99-106. [CrossRef]

44. Chan, T.F.; Kerkhoven, T. Fourier methods with extended stability intervals for KdV. SIAM J. Numer. Anal. 1985, 22, 441-454. [CrossRef]

45. Trefethen, L.N. Spectral Methods in MATLAB; SIAM: Philadephia, PA, USA, 2000.

46. Baqer, S. Dispersive Hydrodynamics in a Non-Local Non-Linear Medium. Ph.D. Thesis, University of Edinburgh, Edinburgh, UK, 2020.

47. Marchant, T.R.; Smyth, N.F. An undular bore solution for the higher-order Korteweg-de Vries equation. J. Phys. A Math. Gen. 2006, 39, L563-569. [CrossRef]

48. Sprenger, P.; Hoefer, M.A. Discontinuous shock solutions of the Whitham modulation equations and traveling wave solutions of higher order dispersive nonlinear wave equations. Nonlinearity 2020, 33, 3268-3302. [CrossRef]

49. Smyth, N.F. Modulation theory solution for resonant flow over topography. Proc. Roy. Soc. Lond. A 1987, 409, 79-97.

50. El, G.A. Resolution of a shock in hyperbolic systems modified by weak dispersion. Chaos 2005, 15, 037103. [CrossRef] [PubMed]

51. Horikis, T.P. Small-amplitude defocusing nematicons. J. Phys. A 2015, 48, 02FT01. [CrossRef]

52. Kodama, Y.; Taniuti, T. Higher order approximation in the reductive perturbation method. I. The weakly dispersive system. $J$. Phys. Soc. Jpn. 1978, 45, 298-310. [CrossRef]

53. Marchant, T.R.; Smyth, N.F. The extended Korteweg-de Vries equation and the resonant flow of a fluid over topography. J. Fluid Mech. 1990, 221, 263-288. [CrossRef]

54. Laudyn, U.A.; Kwaśny, M.; Sala, F.A.; Karpierz, M.A.; Smyth, N.F.; Assanto, G. Curved optical solitons subject to transverse acceleration in reorientational soft matter. Nat. Scien. Rep. 2017, 7, 12385. [CrossRef]

55. Congy, T.; El, G.A.; Hoefer, M.A. Interaction of linear modulated waves and unsteady dispersive hydrodynamic states with application to shallow water waves. J. Fluid Mech. 2019, 875, 1145-1174. [CrossRef]

56. El, G.A.; Khamis, E.G.; Tovbis, A. Dam break problem for the focusing nonlinear Schrödinger equation and the generation of rogue waves. Nonlinearity 2016, 29, 2798-2836. [CrossRef]

57. Marchant, T.R.; Smyth, N.F. Approximate techniques for dispersive shock waves in nonlinear media. J. Nonlin. Opt. Phys. Mater. 2012, 21, 1250035. [CrossRef] 
58. Marchant, T.R. Asymptotic solitons of the extended Korteweg-de Vries equation. Phys. Rev. E 1999, 59, 3745-3748. [CrossRef]

59. Gelfand, I.M.; Fomin, S.V. Calculus of Variations; Prentice-Hall: Englewood Cliffs, NJ, USA, 1963. 